Tageslicht verhalt, musste ich bei einem Versuche, meine Prăparate Demonstration halber zu projiciren, unliebsam erfalren.

Bezüglich der verwendeten Vergrösserung sei noch bemerkt das in ihrer Ganze roth gefärbte, weil in ihrer ganzen Ausdehnung von Mucin durchsetzte Zellen auch bei mittelstarken Systemen sehr gut differenzirt erscheinen; wăhrend an Zellen, die nur in gewissen Theilen Schleim enthalten (z. B. Oberenden der Oberflächen-Epithelien des Magens) der Farbencontrast nur bei stărksten Immersions-Systemen in tadelloser Schärfe hervortritt.

$\mathrm{Za}$ welchen Resultaten ich mittelst dieser verbesserten Schleimfärbung speciell bezüglich des Oberflächen-Epithels des menschlichen Magens gelangte, wird an anderer Stelle ${ }^{1}$ ) ausführlich dargethan. Hier sei nur kurz erwăhnt, dass die SchleimReaction sowohl im erwähnten Oberflächen-Epithel, als auch an den Becherzellen des Magens und Darmes, so wie am Nabelstrang immer eindeutig positiv ausfiel.

A us dem pathologisch-anatomischen Institut der k. ung. Universität zu Budapest. Director Professor Dr. Otto Pertik.

\title{
Ueber das normale Oberflächen-Epithel des Magens und über Vorkommen von Rand- saumepithelien und Becherzellen in der menschlichen Magenschleimhaut.
}

\author{
Von \\ Dr. Pa u 1 Hár $\mathbf{i}$ \\ in Budapest.
}

Hierzu Tafel XXXV und 2 Tabellen.

Wenn die pathologische Anatomie die Grundlage zur Erkenntniss des Wesens der Krankheitsprocesse im menschlichen Körper liefern soll, kann man sich der Befürchtung nicht erwehren, dass es um unser Wissen bezüglich des Wesens der

1) Dieses Archiv, dieser Band, dieses Heft, S. 685. 
Magenkrankheiten ziemlich sehlecht bestellt sei. Und wirklich krankt dieser wichtige Abschnitt der inneren Medicin trotz der bedeutsamen Fortschritte der letzten zwanzig Jahre bis zu einem gewissen Grade noch an dem Uebel, das vor mehreren Decennien der gesammten Medicin gemein war: die Auffassung ersetzt die Erkenntniss, der Terminus deckt den nicht erkannten Thatbestand. Symptome gelten als Krankheitsformen; daher gehen Nomenclatur, Klinik und Therapie der Magenkrankheiten jede ihren gesonderten Weg, als fehlte ihnen die auf objectiver Erkenntniss beruhende gemeinsame Grundlage, d. i. die specielle Pathohistologie des Magens. Diese ist in der That weit davon entfernt, ausgebaut zu sein.

Bei einigermassen exacten pathohistologischen Kenntnissen würden wir auch die Krankheitsprocesse im Magen genauer kennen; wir wären dadurch der Nothwendigkeit enthoben, functionelle Störungen so wie auch den etwa geănderten Chemismus oft als selbstständige Krankheitsformen anerkennen zu müssen und könnten sie einfach als consecutive Erscheinungen jener bis heute nicht genau erkannten Krankheitsprocesse deuten.

Hochgradige postmortale (und theilweise auch agonale) Veränderungen der oberen Schleimhautschichten (besonders der Epithelien) vereiteln die Verwendung des Cadavermagens zu feineren histo- und pathohistologischen Studien. Dies, sowie die Schwierigkeiten beim Erlangen lebensfrischen, respective lebensfrisch fixirten Materials machen die Lücken in unserem Wissen erklärlich.

Boas' anregende Hinweise, sowie mehrere Arbeiten der letzten Jahre, die an solch frischem Material vorgenommen wurden, sind daher um so höher anzuschlagen, als auch deren Ergebnisse genug verheissungsvoll sind, um den von ihren Autoren angetretenen l'fad als den richtigen erkennen zu lassen.

In Nachstehendem soll über Untersuchungen berichtet werden, die an einer grossen Anzahl von lebensfrisch fixirten Magenschleimhaut-Stückchen angestellt wurden, deren Ergebnisse aber hier nur insofern berïcksichtigt sein sollen, als sie sich auf folgende, auch physiologisch wichtige Fragen beziehen:

1. Morphologie und Schleimreaction des Oberflächen- und Vorraum - Epithels;

2. Becherzellen und Randsaum-Epithelien im menschlichenMagen. 


\section{Zur Untersuchung kamen:}

a) Schleimhautfragmente, wie sie durch den weichen Magenschlauch theils aus dem nüchternen Magen, theils nach einem Probefrühstick herausbefördert wurden. (18 Stücke):

b) Grössere Schleimhautstücke, die ich bei Mąenoperationen (Gastrostomie, Gastroenterestomie, Totalexsterpation des Magens) erhielt (13 Fälle), und zum weitaus grössten Theile der Güte des Herrn Docenten Dr. Herczel in Budapest verdanke.

Die Fixation erfolgte zu Beginn meiner Arbeit mittelst Alcohol, später mittelst concentrirter Sublimatlösung (Sublimat 7, Kochsalz 0,5 auf 100 Wasser). Dass die nach letzterer Methode gewonnenen Präparate zum Studium der Verhältnisse des Oberflächen-Epithels weit mehr als Alcohol geeignet sind, soll weiter unten gezeigt werden.

I.

Aus den dargethanen Gründen ist über den Bau des Oberflächenepithels, das im Leichenmagen durch postmortale Verdauung zu allererst zerstört wird, das letzte Wort noch lange nicht gesprochen.

Oppel sagt in seinem grundlegenden Werke ${ }^{1}$ ): „Der Umstand, dass frisches menschliches Material sehr schwer zu bekommen ist und selbst solches sehr häufig pathologische Erscheinungen zeigt, erklärt es, dass die Forschungsergebnisse an Menschen hinter denen bei den Vertebraten zurückstehen. Dies kann aber nicht begründen, anzunehmen, dass hier andere Verhältnisse bestehen ...... oppel unterscheidet an den Zellen des Oberflächenepithels, und zwar sowohl bei sämmtlichen Wirbelthieren im Allgemeinen ${ }^{2}$ ) als auch bei allen Säugern ${ }^{3}$ ) speciell zwei Abtheilungen, welche sich gegen einander scharf absetzen: „einen basalen protoplasmatischen und einen peripheren (der Oberfläche zu gelegenen) ......; letzteren bezeichnet er als Oberende. Wir haben daher allen Grund, anzunehmen, dass es sich im Menschenmagen genau so verhalte. In der That schreibt Ebner hierüber in Koelliker's ${ }^{4}$ ) Handbuch: „Im Allgemeinen kann man an jeder Zelle im frischen Zustande zwei Abtheilungen unterscheiden; eine tiefe, der Schleimhaut auf-

1) Oppel Albert. Lehrbuch der vergleichenden mikroskopischen Anatomie der Wirbelthiere. Erster Theil, 1896. S. 464.

2) 1. c. S. 11 .

3) l. c. S. 219 .

4) A. Koelliker's Handbuch der Gewebelehre des Menschen. VI. Auflage. III. Band von Victor von Ebner. S. 152. 
sitzende .....- und eine oberflächliche, welche etwas dunkler erscheint und von relativ grösseren Körnchen erfüllt ist ......"

E b n e r's Angaben stimmen mit denen 0ppel's so ziemlich überein, namentlich auch darin, dass

1. offene Zellen nur Kunstproducte sein können.

0 ppe $^{1}$ ) schreibt diesbezüglich: „..... Das Oberende zeigt sich bei gut erhaltenen Zellen intact"; d. h. es kommt "unter normalen Verhältnissen nicht zum Ausfliessen derselben, wie manche Autoren annehmen wollten."

Bei Ebner ${ }^{2}$ ) heisst es: „...... Aehnlich wie Schleimzellen sind die Cylinderzellen der Magenoberfläche sehr empfindliche Gebilde, die durch die meisten Reagentien und sehr bald beim Absterben eingreifende Veränderungen erleiden. Die dunkle oberflächliche Abtheilung quillt sehr stark auf, fliesst zum Theil oder ganz aus dem freien Ende hervor. Nun haben die Zellen ein den mit Reagentien behandelten Becherzellen ähnliches Aussehen."

Dies kann ich auch meinerseits vollinhaltlich bestätigen. $\mathrm{Zu}$ den vielen Irrthümern, die diesbezüglich begangen wurden, gaben die Qualitätsmängel der untersuchten Objecte, sowie fehlerhafte Technik, Veranlassung. Denn Schleimbautstïcke, die nicht alsobald nach ihrer Loslösung vom lebenden Magen fixirt wurden, können keine getreuen Bilder über die Verhältnisse des Obertlächen-Epithels liefern; aber auch solche, die sofort in verdünnten oder absoluten Alcohol kamen, sind für diese Studien durchaus unbrauchbar. An solchen Objecten findet man in der That alle möglichen Epithelformen wieder, die von verschiedenen Autoren beschrieben und mannigfach gedeutet wurden: halbleere Zellen, an deren Mündung noch ein in Ausstossung begriffener Propf steht, leere Dütenformen, sowie auch andere leere Zellen, die Becherzellen vortäuschen. Bei Anfertigung von Querschnitten durch die Magenschleimhaut werden die Drüsenvorräume im Profil getroffen, oft derart, dass nebst den Seitenwänden des Drüsenvorraumes auch dessen Rückwand erhalten bleibt. Haben wir es nun mit einem in Alcohol fixirtem Präparat zu thun, so

1) OppeI l. c. S. 11.

2) Ebner l. c. S, 152 . 
bietet dasselbe das allbekannte, vielfach reproducirte, netzartige Bild mit leeren oder fast leeren Maschen (Fig. 1 u. 13). Von der Zellsubstanz sieht man nur spärliche, geschrumpfte Reste, die dem Balkenwerk des Netzes seitlich sich anschmiegen. Für viele Autoren lag nun die Deutung nahe, dass der Zellinhalt, zumindest des Oberendes, bereits in Form voll Schleim ausgestossen ward, und nunmehr die Zellwände (verdicktes Ectoplasma) als leeres oder fast leeres Maschenwerk übrig blieben.

Man vergleiche hiermit das Bild eines mit Sublimat fixirten Schleimhautstückchens (Fig. 2). Statt der oben beschriebenen, durch Schrumpfung entstellten Formen, sind hier nur succulente vorzüglich erhaltene Zellen sichtbar, an denen das von Oppel sogenannte Oberende mit grösster Deutlichkeit nachweisbar ist und statt des leeren Netzes sieht man ein wohlangefülltes Mosaik, gebildet durch jene succulente Zellen.

Am besten eignet sich combinirte Hämatoxylin-EosinFärbung; nur soll man die wässerige Eosinlösung nur ganz kurz einwirken lassen, da sonst der ganze Zellleib nahezu homogen roth erscheint. Bei gelungener Färbung erscheint das Oberende hell rosenroth, der basale Theil aber dunkelroth.

Thioninfärbung eignet sich mehr zum Nachweise des chemisch differenten Verhaltens beider Zellabtheilungen als zur Darstellung der scharfen Grenze zwischen denselben.

2. An solchen Hämatoxylin-Eosin-Präparaten grenzt sich das Oberende gegen das basale Ende in einer nach oben concaven Bogenlinie scharf ab, genau wie dies oppel sogar von einer Fischart, Raja asterias, abbildet (Fig. 22).

Ebner sieht am frischen Objecte $^{1}$ ), speciell auch im Salamandermagen ${ }^{2}$ ) keine scharfe Grenze zwischen beiden Zellabtheilungen.

Dies soll für lebensfrische Objecte, aber nur für diese, auch zugegeben sein; mit der Beschränkung jedoch, dass, wenn auch diese scharfe Grenze an der lebensfrischen Zelle zwar nicht sichtbar ist, man nicht behaupten kann, dass sie nicht vorhanden sei. Diesen meinen Standpunkt möchte ich durch Folgendes begründen:

1) 1. c. S. 152 .

$\Rightarrow$ ) l. c S. 154 . 
Das in Frage stehende Oberende schliesst, wie dies am gehărteten, gefärbten und aufgehellten Schnitte zu sehen ist, mit einer, nach oben concaven, Linie nach unten ab. Auf körperlose Dimensionen bezogen, heisst dies soviel, dass das Oberende nach unten etwa eiförmig abgerundet endigt, das Basalende aber entsprechend eiförmig ausgekehlt ist. Diese beiden Theile sind nun dermassen ineinander gepasst, dass der eingeschlossene untere Pol des Oberendes eine deckende Hülse aus basaler Substanz erhält, die, nach oben an Dicke abnehmend, noch vor dem freien Zellende aufhört' ${ }^{1}$ ).

An der lebensfrischen Zelle, die als geschlossener Körper (also nicht angeschnitten wie unsere Dauerpräparate) quasi in Vogel-Perspective betrachtet wird, kann man daher das Oberende, das mit gröberen Körnchen erfüllt und dunkler ist ${ }^{2}$ ), vom basalen Theil wohl unterscheiden, ohne aber zwischen beiden eine scharfe Grenze zu sehen. Denn einerseits werden die Conturen des unteren Poles des Oberendes durch eine hier noch dicke Schicht der deckenden basalen Substanz undeutlich gemacht, anderseits wird aber auch die Stelle, wo die schon ganz dünn gewordene Hülse aus basaler Substanz allmählich endet, über dem dunklen hier schon dicken Oberende nicht zu sehen sein.

Anders an den Schnittpräparaten: Hier werden die Zellen nicht als geschlossene Körper, sondern insoferne als sie auf den Firsten sitzen oder die Seitenwănde der Drüsen-Vorräume bekleiden (in der Regel der Länge nach) aufgeschnitten betrachtet. Hier ist die Uebergangsstelle, richtiger die Trennungslinie von keiner anderen Schicht bedeckt und in Form der oben mehrmals erwăhnten concaven Linie auf das Schärfste wahrnebmbar.

Dort wo diese Zellen nicht der Länge nach sondern quer getroffen sind und das ist an der Rückwand der DrüsenVorräume der Fall, entstehen Bilder, die obige Verhältnisse deutlich illustriren. - Fällt die Schnittebene schon ausserhalb (oberhalb) des Bereiches der basalen Hülse, erscheinen runde bis polygonale Scheiben, die genau so gefărbt sind, wie die Oberenden an den

1) Ich gebrauche diesen Vergleich, um mich besser verständlich zu machen, ohne dabei mehr sagen zu wollen, als dass ein Uebergang zwischen beiden Abschnitten zwar zweifelsohne besteht, jedoch so schnell erfolgt, dass er, auch mit Immersions-Systemen betrachtet, als scharfe Linie erscheint.

*) E b a e r I. c. S. 152. 
Långsschnitten; wurden aber die Zellleiber etwas weiter gegen das basale Ende zu getroffen, wo noch eine wenn auch dünne Hülse aus basaler Substanz vorhanden ist, sehen wir als Profilbild der neben einander gereihten Hülsen ein dünnbalkiges Maschenwerk, das genau so gefärbt ist wie die basalen Enden der Zellen; in die Lücken dieses Maschenwerks sind die runden Scheiben (in den Farben der Oberenden) aufgenommen.

Ohne weitere Belege anführen zu wollen oder zu können, halte ich es nicht für ausgeschlossen, dass das, was als seitliche Zellmembran ${ }^{1}$ ) (oder verdicktes Ectoplasma) des Oberflächenepithels vielfach beschrieben wurde, im Wesentlichen diesen Hülsen aus basaler Substanz entspricht.

3. Was die Conturen namentlich am freien Ende des Oberflaichenepithels anbelangt, fand ich genau dieselben Verhältnisse, wie dies Ebner bezüglich der lebensfrischen Zelle ${ }^{2}$ ) beschreibt: „Das freie Ende der Zelle ist entweder fast gerade abgestutzt oder hervorgewölbt." - Schnitte von in Sublimat fixirten Objecten weisen beide Formen auf (Fig. 2, 14, 15, 16), die übrigens nichts wesentlich Verschiedenes haben. Aus dieser Uebereinstimmung des Bildes der lebensfrisch und in Sublimat fixirten Zelle geht wieder hervor, dass Sublimat durchaus geeignet ist, die Zellen in ihrer wahren Form zu fixiren; daher man auch füglich annehmen kann, dass an den Zellen, deren aussere Conturen unverändert erhalten sind, auch die scharfe Grenze zwichen basalem und Oberende nicht als Kunstproduct angesehen werden darf.

4. Betreffs der räumlichen Ausdehnung des basalen Theiles und des Oberendes innerhalb eines Zellleibes sagt Ebner, dass die dunklere Abtheilung (Oberende) bald grösser bald geringer sein kann $^{3}$ ). - Dies entspricht genau dem, was ich an meinen Präparaten sah. Bald fand ich das Oberende auf das oberste Viertel oder Fünftel der Zelle beschränkt (Fig. 2), bald nahm es die halbe oder gar fast die ganze Höhe der Zelle ein, (Fig. 15, 16) sodass das protoplasmatische basale Ende den immer grossen, eventuell auch platt gedrückten Kern, nur in dünner Schicht umgab.

ग) Oppel. 1. c. S. 11 .

z) Ebner. l. c. S. 152.

s) Ebne r. 1. c. S. 152. 
Von hohem Interesse ist es, hiermit zu vergleichen, was Oppel bezüglich des Oberflächen-Epithels des Fischmagens schreibt: „Bei verschiedenen Fischen ist verschieden: einmal die Grösse der Epithelzellen, die Grösse des basalen (protoplasmatischen) Theiles und die Grösse des Oberendes, endlich Grösse und Lage des Kernes" . . "Auch bei ein und demselben Thiere zeigen die Zellen kleine Verschiedenheiten. Doch nicht so, dass etwa eine Zelle mit grossem Oberende neben einer solchen mit kleinem Oberende stände, vielmehr sind die Uebergănge stets ganz allmåhliche ..." Da liegt die Frage nahe, ob es wohl angeht, aus der grösseren Ausdehnung des Oberendes (das als verschleimte, richtiger schleimführende Zellabtheilung aufgefasst wird) auf einen pathologischen Verschleimungsprozess zu folgern, wăhrend doch ăhnliche Bilder, wie wir soeben sahen, auch bei Fischen zur Regel gehören, die doch gewiss nicht magenleidend sind!

Auf diese Frage wollen wir nach Besprechung der chemischen Beschaffenheit und der mikrochemischen Farbenreaction des Oberendes zurückkehren.

5. Bezüglich des Schleimgehaltes des Oberendes des Oberflächenepithels im Wirbelthiermageu sagt oppel ${ }^{2}$ ) und Rawitz ${ }^{3}$ ), weiterhin Oppel vom Fischmagen ${ }^{4}$ ) und vom Saugethiermagen ${ }^{5}$ ), dann Bannwarth und Cremer $^{6}$ ), dass die Oberenden wohl nicht ausschliesslich aus Schleim bestehen, jedenfalls aber Schleim oder schleimähnliche Substanzen enthalten.

Vom menschlichen Magen sagt 0 p pel ${ }^{7}$ ), dass: „.... die Forschungsergebnisse am Menschen hinter denen bei den Vertebraten zurückstehen. Dies kann aber nicht begründen, anzunehmen, dass hier andere Verhältnisse bestehen. ...."

1) Oppel l. c. S. 36 .

2) 0 ppel l. c. S. 11 .

3) citirt bei Oppel l. c. S. 16.

4) 1. c. S. 36 .

5) l. c. S. 219 .

6) citirt bei Oppel 1. c. S 222 und 223. Bannwarth, E., Histologie Leipzig 1894 und Cremer Werner, Untersuchungen über die chemische Natur des Schleimkörpers der Magenschleimhaut. Inaug. Diss. Bonı 1895. i) l. c. S. 464 . 
Ho ye ${ }^{1}$ ) sagt: Das Secret, das die Zellen des Oberflächenepithels erfüllt, wird .... von macinfärbenden Lösungen durchaus nicht tingirt (auch nicht ron Carmin). Trotzdem dürfte dieselbe dem Mucin sehr nahe stehen ...."

Aehnliches sagt auch W a r bur $g^{2}$ ).

Creme $\mathrm{r}^{\mathrm{g}}$ ) concludirt dahin, dass die Oberflächenepithelien im menschlichen Magen keine echte Mucinreaction zeigen.

Cohnheim ${ }^{4}$ ) kann sich auf Grund seiner Befunde der Ansicht Stöhr's nicht anschliessen, welche die schleimige Beschaffenheit der freien Enden der Cylinderepithelien für normal hält.

Schmidts) muss $n . .$. die Verschleimung des Protoplasmas im äusseren Ende als eine inhärente Eigenschaft des Magenepithels ansehen, die auch .... in dem Magen des fünfmonatlichen Embroyo .... bereits deutlich ausgesprochen war....

Nach L ubarsch's Ansicht ${ }^{\theta}$ ) ist die ${ }_{n}$ Verschleimung. . . . ein normaler Vorgang, den man in ganz normalen Magen niemals vermisst, der aber mitunter auf die äusserste Peripherie der Zellen beschränkt ist."

$S c h$ affe $r^{7}$ ) kommt ${ }_{n} z u$ einem ähnlichen Resultat; er hebt von den Magenschleimzellen ${ }^{8}$ ) hervor, "dass Dela field's Hämatoxylin-Thonerde, Mucicarmin etc. die Zellen intensiv färbt, wenn sie nur frisch genug zur richtigen Fixirung gelangen."

Ebenso betont $\mathrm{E} b \mathrm{n} \in \mathrm{r}^{9}$ ) die schleimige Natur des Oberendes, das „.... mit den specifischen Schleimfärbemitteln (Mucicarmin etc.) sich färben lässt; freilich nicht so intensiv wie die Zellen der Schleimdrüsen oder Becherzellen."

Die Ansichten der Autoren sind nach dem Angeführten in dieser Frage ziemlich divergirend. Am schroffsten nehmen Cremer und Cohnheim gegen die schleimige Natur, respective

1) H. Hoyer. Ueber den Nachweis des Mucins in Geweben vermittelst der Färbemethode. Archiv für microscopische Anatomie. Band 36. 1890.

2) Citirt bei 0 p pe l. 1. c. S. 222.

3) Citirt bei oppel. l. c. S. 223.

4) Cohnheim, Paul. Die Bedentung kleiner Schleimhautstückchen für die Diagnostik der Magenkraukheiten. Archiv für Verdauungskrankheiten. Band 1, Heft 3. S. 281.

$\left.{ }^{5}\right)$ Adolf Schmidt. Untersuchungen über das menschliche Magenepithel unter normalen und pathologischen Verhältnissen. Virchow's Archiv für pathologische Anatomie und Physiologie, Band 143. S. 483.

$\left.{ }^{6}\right)$ F. Martius. Achilia gastrica mit einem anatomischen Beitrage von 0. Lubarseh. 1897. S. 132 .

7) Josef Sch affer. Beiträge zur Histologie menschlicher Organe. Aus den Sitzungsberichten der kaiserlichen Akademie der Wissenschaften in Wien. Abth. III. October 1897.

$\left.{ }^{8}\right)$ D. h. die Zellen des Oberflächenepithels.

9) Ebner. 1. c. S. 153.

Archiv f. mikrosk. Anat. Bd. 58. 
gegen die echte Mucinreaction der Oberflächen-Epithelien Stellung, während die Mehrzahl neuerer Autoren direct dafür eintritt.

In dem von Hoyer 1890 näher beschriebenen und als Schleim-Reagens anempfohlenen 'Thionin ${ }^{1}$ ) fand ich ein vorzügliches Mittel, um diese Frage zu entscheiden und zwar gelang mir dies (meines Dafürhaltens) im Sinne des sicheren Schleimgehaltes, respective der echten Mucin-Reaction; jedoch erst, als ich eine von mir an anderer Stelle ${ }^{2}$ ) beschriebene Modification der Hoyer'schen Methode fand.

Das Ergebniss der Färbung mittelst dieser Metode war ein schwankendes, insolange es sich um Alcoholpräparate gehandelt hat; nach dem, was ich über die Nachtheile der Alcoholfixation weiter oben mitgetheilt habe, ist dies auch leicht verständlich: In dem Maasse, als die schleimhaltigen Oberenden im Alcohol mehr oder weniger zusammengeschrumpft waren, ist auch die Thionin-Reaction weniger oder mehr distinct ausgefallen; ich besitze Schnitte von zwei in Alcohol fixirten Schleimhautstückchen, die durchwegs vorzügliche Resultate gaben (Fig. 4 und 5), andere, bei denen man Mühe hat, einzelne gute Stellen zu finden.

Insgesammt handelt es sich um 15 Alcoholpräparate, von denen 5 deutliche Schleimreaction gaben.

Anders an den mit Sublimat fixirten, von denen mir 16 Stücke zur Verfügung standen; bei diesen konnte ich in den schleimführenden Theilen, d. h. in den Oberenden ohne Ausnahme jedesmal sicher Schleim nachweisen.

Wie in den Fig. 14, 15 und 16 sichtlich, färbten sich die Oberenden violett-roth, während die übrigen Gewebselemente, und namentlich auch die basalen Theile der Zellen des Oberflächenepithels blau blieben. Dass dieses Roth einmal heller (Fig. 16) ein anderesmal dunkler (Fig. 14, 15) ausfällt, ist wohl selbstverständlich; denn von anderen, weiter unten zu erörternden Umständen abgesehen, werden begreiflicherweise Färbung und Entfärbung, die so viele Phasen aufweisen, kaum vermeidliche Schwankungen in der Intensität der Endreaction zeitigen.

Die 16 mit Sublimat fixirten Objecte, die ohne Ausnahme positive Schleimreaction der Oberenden gaben, stammten theils

1) 1. c

2) Dieses Archiv, dieser Band, dieses Heft. S. 678 . 
von solchen Fällen ab, die einen clinisch (auch chemisch) vollkommen normalen Magenbefund boten; theils von solchen, die an den verschiedensten Erkrankungen litten: Hypersecretion, Hyperchlorhydrie, Gastritis acida, Carcinoma ventriculi, Carcinoma oesophagi, narbige Oesophagus- und Pylorus-Stenose, alte UlcusNarbe der kleinen Curvatur.

Diese Objecte liessen bald mit grösster Deutlichkeit ibre Zugehörigkeit zum Fundus- oder zum Pylorus-Theile erkennen, bald war eine solche Ursprungs-Bestimmung nicht möglich; Drüsen und interstitielles Gewebe boten in einer Reihe der Fälle keine Spur einer pathologischen Veränderung, waren in anderen Fällen hochgradig verändert; - das Verhalten des Oberflächenepithels blieb immer dasselbe.

Es ist hieraus zu ersehen, dass der Schleimgehalt der Oberenden eine inhärente Eigenschaft des Oberflächen-Epithels ist, ob nun dasselbe aus einem kranken oder gesunden Magen stammt.

Ich muss wohl zugeben, dass ich Präparate besitze, an denen die grossen und ganz grossen Oberenden überwiegen (Fig. 3); andere, die viele kleine Oberenden aufweisen (Fig. 2); immerhin sind bei jenen kleine, bei diesen grosse Oberenden doch in grosser Zahl anzutreffen.

Ich kann also einen wesentlichen Unterschied, geschweige denn einen typischen Befund nach keiner der beiden Richtungen hin (wenig oder stark verschleimt) statuiren; umsoweniger, da eine Coincidenz mit den pathologischen Veränderungen der übrigen Schleimhautelemente an den betreffenden Schnitten oder mit dem klinischen Befund (etwa Schleimgehalt des Mageninhaltes) nicht $\mathrm{zu}$ constatiren war.

Es ist möglich, dass Verdauungs-Phase ${ }^{1}$ ) oder Provenienz des Schleimhautstückchens (ob Fundus oder Pylorustheil) Unterschiede in der Ausdehnung des Oberendes bedingen, was aber erst durch weitere Untersuchungen klargestellt werden müsste.

Es variirt aber nicht nur die räumliche Ausdehnung, sondern augenscheinlich auch der Schleimgehalt der Oberenden und zwar vielleicht wieder den Verdauungsphasen entsprechend. Lehrreich sind in dieser Beziehung Bilder von Drüsenvorräumen, in deren

1) Wilhelm Ebstein. Beiträge zur Lehre rom Bau und den physiologischen Functionen der sogenannten Magenschleimdrüsen. - Archiv für microscopische Anatomie Band VI, 1870, S. 515. 
Lumen ein Schleimpfropf von meistens etwas gewundenem Verlaufe sitzt; bald erscheinen nămlich Oberenden und Schleimpfropf, d. i. das reine Secret der ersteren, durch Thionin fast gleich stark gefärbt, bald fällt die Reaction am Pfropf, weil dieser eben reines Secret darstellt, viel intensiver aus (Fig. 3).

Diese Unterschiede in der Färbung sind auch leicht verstăndlich, wenn man den Inhalt des Oberendes sich so vorstellt, dass ein Gerüste aus protoplasmatischer (?) Substanz variable Mengen schleimigen Secrets enthalt.

Wir begegnen bei den verschiedensten Autoren dem Ausdruck: Verschleimung des Oberflächen- und Vorraum-Epithels; und zwar handelt es sich das einemal um einfache Verschleimung der Cylinderzellen, ein anderes Mal um Verschleimung mit Bildung von Becherzellen und Randsaum-Epithelien. Dass Becherzellen und Randsaum-Epithelien mit einem pathologischen Processe, also auch mit Verschleimung, durchaus nichts zu thun haben, soll weiter unten gezeigt werden. Hier reflectire ich nur auf eine Verschleimung der Cylinderzellen, die ich nach Obigem, im Sinne einer pathologischen Veränderung, nicht zugeben kann, womit ich natürlich nicht in Abrede stellen will, dass die Oberenden, als schleimbildende Organe des Oberflächenepithels, in einem Falle wenig, in einem anderen aber überreichlich Schleim secerniren, ohne aber diese verminderte oder gesteigerte Thätigkeit an ihrer Form oder Grösse erkennen zu lassen. Analoga aus der Reihe anderer Drüsenzellen brauche ich hierfür wohl nicht anzuführen.

Bereits 1897 wendet sich Lubars chl) gegen $\mathrm{P}$. Cohnhei m's Befunde, die sich auf verschleimte Cylinderepithelien, als pathologische Veränderungeu beziehen: ,Wenn $\mathrm{P}$. $\mathrm{Coh} \mathbf{n h}$ e i m ........ mehrfach von einfachem, nicht verschleimtem Cylinderepithel spricht und im Gegensatz dazu das Vorkommen am freien Ende verschleimter Cylinderepithelien als einen pathologischen Vorgang ansieht, so ist mir das nicht ganz verständlich; entweder bezeichnet er nur solche Zellen als verschleimt, die die echte Schleimreaction (Thionin-, Methylgrün-Färbung) geben, oder er hat infolge ungenügender Färbung die Schleimpartikelchen im Oberende vermisst. Dass das Vorkommen von gewöhnlichem, mit Methylgrün färbbarem Mucin etwas pathologisches ist, muss ich allerdings auch anerkennen."

Dem ersten Theil dieser L u b a r s c h'schen Conclusion schliesse ich mich vollständig an. Versteht aber Lubarsch unter "gewöhnlichem" Schleim auch solchen, der mit T'hionin sich

1) l. c. S. 132 . 
färbt und hält er dessen Vorkommen für pathologisch, so muss ich dies auf Grund des oben ausgeführten als nicht zutreffend bezeichnen. Das Verhalten des Oberflächenepithels zu Methylgrün ist mir nicht bekannt.

Meine Schluss-Conclusionen lauten daher:

Die Zellen des Oberflächenepithels und der Drüsenvorräume am menschlichen Magen sind nach dem allgemeinen Wirbelthier-und speciell auch nach dem Säugethier-Typus gebaut; sie bestehen aus dem kernhaltigen basalen und dem von Oppel sogenannten Oberende, das mikrochemisch nachweisbar Schleim enthält. Daher man von einer Verschleimung der Epithelzellen als von einer pathologischen Veränderung nicht sprechen kann.

II.

\section{Becherzellen und Randsaum-Epithelien im menschlichen Magen.}

Seit den diesbezüglichen Funden von $K$ upffer ${ }^{1}$ ), Ewald ${ }^{2}$ ) und Anderen mehren sich namentlich seit 1895 die Angaben verschiedener Autoren über das Auftreten von Becherzellen und Randsaum-Epithelien im menschlichen Magen. Diese Befunde sind in der That sehr eigenartig und werden in der Regel als Zeichen einer schleimigen Entartung der Magenepithelien aufgefasst.

Da sowohl die einschlägigen literarischen Angaben, wie auch die Ansichten bezüglich der Aetiologie und diagnostischer Verwerthbarkeit dieser Bilder zahlreich und vielfach widersprechend sind, wird es nöthig sein, einen kurzen Ueberblick über die erwähnten Befunde zu geben, erst aber genauer zu präcisiren, um was es sich eigentlich handelt.

An Schnitten aus manchen Magenschleimhaut-Stückchen zeigt das Oberflächen-Epithel und das der Drüsenvorräume stellenweise einen vom gewöhnlichen durchaus verschiedenen Character.

Während für gewöhnlich einander durchwegs gleichende cylindrische Zellen aneinander gereiht sind, die Zelle für Zelle

J) C. Kupffer. Epithel und Drüsen des menschlichen Magens. Abdruck aus der „Festschrift des ärztlichen Vereins München“. München 1883;

2) Ewald. Klinik der Vordauungskrankheiten. 
ein schleimführendes Oberende besitzen, sehen wir in einer anderen Reihe von Fällen das Epithel der Oberfläche und der Drüsenvorräume auf kürzere oder grössere Strecken den Character des Darmepithels bis ins letzte Detail nachahmen: schlanke cylindrische Zellen ohne schleimführendes Oberende, dagegen mit deutlichst gestricheltem oder gar zerfasertem Randsaum werden in regelmässigen Intervallen von typischen Becherzellen unterbrochen, die ihrerseits exquisiteste Schleimreaction geben. (Siehe hierüber die betreffenden Abbildungen im ersten Theil des Boas'schen Lehrbuches). Immer ist die Grenze zwischen den beiden Epithelarten, also dem erst beschriebenen Magen- and dem zuletzt beschriebenen typischen Darmepithel eine ganz scharfe.

Die Ku pffer'schen Angaben über das Vorkommen von becherzellen in dem von ihm beschriebenen Magen Nr. $3^{1}$ ) sind nicht ganz eindeutig: "In sämmtlichen Regionen dieses Magens zeigt das Epithel typische Becherzellen. . . . . Man findet ausserdem alle Zwischenstufen zwischen unveränderten Zellen und stark aufgeblähten."

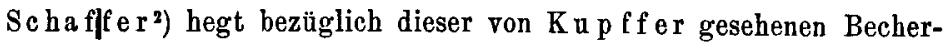
zellen berechtigte Bedenken, namentlich weil dieser sie in nsämmtlichen Regionen" beobachtet haben will. Der Nachsatz "alle Zwischenstufen" ist noch mehr geeignet, Bedenken zu erregen.

Wie schwankend übrigens der Begriff „Becherzellen" selbst im Jahre 1890 noch war, geht aus einer Aeusserung Kle in' ${ }^{3}{ }^{3}$ hervor, der von den Epithelzellen des Magens behauptet, dass „die meisten mucinsecernirende Becherzellen sind."

Sehr richtig ist, was 0 p pel ${ }^{4}$ ) diesbezüglich sagt: ${ }_{n} . . . . \operatorname{mag}$ mancher Autor für die Cylinderepithelien des Magens den Namen „Becherzellen" gebraucht haben, ohne dass er deshalb die Besouderheit dieser Zellen verkannte und vielleicht ohne dass er sie deshalb wahren Becherzellen gleichstellen wollte."

Dass in Ku p f er's Magen Nr. 3 unter den vielen falschen auch echte Becherzellen gewesen sein mögen, geht aus Abbildung 2 auf seiner Tafel I hervor, wo zwei Epithelien ohne Oberende mit exquisitem Randsaum deutlich wiedergegeben sind, wenn auch ihrer im Text nicht ausdrücklich Erwähnung geschieht. Diese Randsaumepithelien nun kommen ohne echte Becherzellen nicht vor.

Vielfach werden von späteren Autoren die von $K u p f f e r$ als „einfache Schleimdrüsen" beschriebenen Gebilde mit den in Frage stehenden in näheren

1) 1. c. S. 16 .

2) 1. c. S. 88 .

3) Klein, E. Grundzüge der Histologie 2. Auflage 1890, eitirt bei Oppel l. c. S. 221 . - Wie Klein in der 3. Auflage seines Buches, das mir nicht zur Verfügung stand, hierüber denkt, weiss ich nicht.

) l. c. S. 11 . 
Zusammenhang gebracht, daher ich seine diesbezüglichen Worte ${ }^{1}$ ) hier citire: ,. . . . . In eine Magengrube mündet ein Sehlauch von der Gestalt einer Fundusdrüse. . . . . . Der ganze Schlauch wird von dem Cylinderepithel der Oberfläche ausgekleidet. Ich fand solche einfache Schleimdrüsen nur in den mittleren Regionen bei verschiedenen Magen."

G. Meyer) gab bereits 1889 eine vorzügliche Beschreihung und Abbildung der Darmepithelschläuche, die er in zwei Fällen beobachtete.

S achs sah bei seinem Fall $A^{3}$ ) eigenthümliche Drüsen, die meist zu 2-3 im normalen Gewebe sassen und hält dieselben für identisch mit den Darmschleimdrüsen der unteren Darmabschnitte; giebt ihnen daher den Namen Magenschleimdrüsen. Sie sollen übrigens nicht in Pylorus allein sondern auch im Fundus vorkommen.

Mangels einer Abbildung ist der Versuch einer Identificirung der von $\mathrm{Sach}$ s geschilderten Gebilde mit den in Frage stehenden sehr schwer: Die „am freien Ende augenscheinlich eine Membran besitzenden Zellen " entsprechen offenbar unseren Randsaumepithelien. Zutreffend ist auch, dass diese Gebilde zu zwei bis drei gruppirt im übrigen Gewebe erscheinen, so wie auch die Betonung der scharfen Grenze zwischen beiden Epithelarten.

Was die Bedeutung obiger Gebilde anbelangt, so hält sie Sachs: ". . . . für ein pathologisches Product, ausgehend von den einfachen „Schleimdrüsen $K$ u p ff ers.“

lch werde weiter unten beweisen, dass es nicht möglich ist, diese Auffassung, die von $\mathrm{Sachs}$ angefangen, wie ein rother Faden durch die meisten späteren Publicationen sich zieht, in Einklang zu bringen mit dem, was Sachs selbst mittheilt.

In dem von Adolf Schmidt4) 1895 beschriebenen Fall handelt es sich zweifels ohne um die in Frage stehenden Epithelformen; am betreffenden Stück aus der Pylorusschleimhaut sah er: ". . . . . . . . ein stäbchensaumtragendes niedriges Epithel, welches eine ausserordentliche grosse Anzahl von stark secernirenden Becherzellen enthält." Ihre Bedeutung betreffend hält sie Schmidt, wenigstens in dieser Publication, nicht für pathologische Gebilde: es soll vielmehr „.. . . . um ein Erhaltenbleiben besonderer auch normaler Weise im Magen vorkommender Epithelschläuche . . . “d. i. der Kupffer'schen einfachen Schleimdrüsen sich handeln, mit denen Schmidt sie für identisch hält. Dies ist fur mich durchaus unverständlich, da ein Blick auf die $\mathrm{Kupffer}$ 'schen Abbildungen genügt, um zu sehen, dass es dort weder Becher- noch Randsaumzellen giebt.

1) 1. c. S. 17.

2) G. Me y er. Zur Kenntniss der sogenannten "Magenatrophie“. Zeitschrift für clinische Medicin. Band 16, 1889, S. 376.

a) Albert Sachs. Zur Kenntniss der Magenschleimhaut in krankhaften Zuständen. Archiv für experimentelle Pathologie und Pharmocologie. Band 24, S. 118.

4) Adolf Schmidt. Ein Fall von Magenschleimhautatrophie nebst Bemerkungen über die sogenannte ,schleimige Degeneration der Drüsenzellen des Magens." Deutsche medic. Wochenschr. Nr. 19, 1895 
Dass Schmidt diesen seinen Standpunkt später vollständig aufgegeben hat und die Zahl jener vermehrt, die die gesagten Epithelschläuche als pathologische Bildungen ansehen, wird an betreffender Stelle gezeigt werden.

Hammerschlag') und nach ihm Cohnheim ${ }^{2}$ ) sind der Ansicht, dass diese Schläuche den im Sinne Hayem's krankhaft verlängerten und erweiterten Drüsenvorräumen entsprechen. Bevor ich zur Besprechung der gründlichen und inhaltsreichen Cohnheim'schen Arbeit übergehe, sei es mir gestattet, zunächst den Irrthum richtig zu stellen, den Cohnheim ${ }^{3}$ durch zweimalige Nennung der Ebste in'schen Magenschleimdrüsen begeht und der durch die irreleitende Neigung der Autoren, eigene Nomenclaturen zu schaffen, erklärlich ist. E bs te in bezeichnete 1870 sämmtliche Drüsen der Regio pylorica schlechtweg als Schleimdrüsen oder einfache Pepsi ndrüsen (im Gegensatze zu den zusammengesetzten Pepsindrüsen des Fundus, die Haupt- und Belegzellen enthalten). Während Sachs die von ihm gesehenen Gebilde als Magenschleimdrüsen benennt, bezeichnen spätere Autoren die specifischen Drüsen der Regio pylorica, also die Ebstein'schen Schleimdrusen als zusammengesetzte Schleimdrüsen im Gegensatze zu den Kupfer'schen einfachen Schleimdrüsen.

Wenn sich nun Cohnheim gegen Schmidt wendet, der die fraglichen Gebilde, wie wir oben gesehen haben, mit den Kupffer'schen e inf a che n Sch le im drüsen identificirt, kann es sich ja nur um diese, keineswegs aber um die Ebstein'schen handeln. Dies zur Richtigstellung des Irrthums.

Cohnheim's Material bestand aus 31 Magenschleimhautstückchen, welche im Laufe der Jahre auf der Boas'schen Poliklinik gesammelt wurden; hiervon stammten 14 von solchen Patienten her, deren Mageninhalt freie Salzsäure aufwies, 17 aber von solchen, bei denen freie Salzsäure fehlte. Bei der ersten Reihe (mit Salzsäure) kamen Becherzellen nur ein bis zweimal, Randsaumzellen gar nicht vor; dagegen beide in grosser Menge bei den 17 Fällen ohne freie Salzsäure. An der Richtigkeit dieser Beobachtung ist nach der vorzüglichen Beschreibung nicht zu zweifeln und es kann nicht Wunder nehmen, wenn die Coincidenz des Salzsäuremangels mit dem nachweisbaren Auftreten der eigenartigen Schläuche in ursächlichen Zusammenhang gebracht wird. Wo der Fehlschluss liegt, erkläre ich im weiteren Verlauf dieser Arbeit.

Schmidt) nimmt bereits im nächsten Jahre nach dem Erscheinen seiner und der Cohnhei m'schen Arbeit einen wesentlich geänderten Standpunkt ein. Diesmal fand er die Schläuche in 13 von 23 pathologisch ver-

1) Hammerschlag, Albert. Zur Kenntniss des Magencarcinoms. Wiener Klinische Rundschau. 1895. No. 23-26; und: Untersuchungen über das Magencarcinom. II. Theil. Archiv für Verdauungskrankheiten. Band II. 1896.

3) 1 . c.

3) l. c. S. 292 .

4) Sch midt. 1896. l. c. 
änderten Magen. In der Polemik gegen $S a c h s$, der die Schläuche von den Kupffer'schen einfachen Schleimdrüsen abstammen lässt, vergisst er vollkommen ${ }^{1}$ ), dass er 1895 die beiden sogar identificirt hat und erklärt' ${ }^{2}$ ) ihr Auftreten aus der Fähigkeit einzelner Magenepithelien ${ }_{n}$ sich unter pathologischen Verhältnissen in Darmepithelien umzuwandeln " -

Das Auftreten der Darmepithelschläuche hat $\mathrm{Sch} \mathrm{m}$ i d t zweimal auch in der Umgebung von Ulcusgeschwüren beobachtet. ${ }^{3}$ ) Die Richtigkeit dieser Beobachtung mag zugegeben sein und anf ihre Bedentung noch reflectirt werden; durchaus unzutreffend finde ich aber Schmidt's Berufung auf Hauser's $s^{4}$ äbnliche Fälle; denn in der ganzen 78 Seiten starken Abhandlung Hauser's wird von Becherzellen und Randsaumepithelien ke in Wort erwähnt, es handelt sich immer bloss um das Auftreten atypischer bald sehr hoher, bald eher cubischer Epithelzellen.

Ebensowenig ist von Darmepithelschläuchen die Rede bei Griffini und Vassale ${ }^{5}$ ), sowie anch Poggi ${ }^{\beta}$ ), die die Ungebung heilender Magenwunden untersuchten.

L u barsch stand ebenfalls ein reichliches Material zur Verfügung, und zwar 20 Stücke von 11 Patienten mit totalem Salzsäuremangel. Er fand Becherzellen und Randsaumepithelien in sechs Stücken, Becherzellen allein in weiteren vier Fällen. - Auch $L u b a r s c h$, der ,die Aehnlichkeit der veränderten Magenschleimhaut mit Darmschleimhaut" besonders betont, ist $^{7}$ ) der Ansicht, dass es sich um eine pathologische Umwandlung des Vorraumepithels im Darmepithel handelt.

Lubarsch beschreibt ${ }^{8}$ ) ein Präparat, an dem "sogar neben Belegund Hauptzellen zwei deutliche Becherzellen in einer Drüse vorhanden" waren. Diesem ganz vereinzelten Befunde glaube ich keine besondere Bedeutung zumassen zu dürfen; denn zwei zufällig hydropisch veränderte Zellen können sehr wohl becherförmig aufgetrieben sein, ohne irgend etwas mit echten Becherzellen zu thun zu haben, die im Magen sowohl als auch im Darm immer in typischer Abwechslung mit Randsaumepithelien vorkommen.

S chaf fer warnt ${ }^{9}$ ) auch davor, , jede Schleimvacuolenbild ung in einerDarmepithelzelle mit Becherzellenbildung in Zusammenhang zu bringen. . . . Nur wird man die gelegentliche Verschleimung einer Zelle, wodurch ein becher-

1) 1. c. S. 497 .

2) 1. c. S. 499 .

s) 1. c. S. 503 .

4) Ha u s er, Gus ta v. Das chroniscbe Magengeschwür etc. Leipzig 1883.

s) Griffini, I. und Vassale, G. Ueber die Reproduction der Magenschleimhaut. Beiträge zur pathologischen Anatomie und zur allgemeinen Pathologie. Band III. 1888.

$\left.{ }^{6}\right)$ Poggi, Alphonse. La cicatrisation immédiate des blessures de l'estomac en rapport avec les duers modes de susure. Beiträge zur pathologischen Anatomie und zur allgemeinen Pathologie. Band III. 1888.

7) 1. c. S. 133 .

8) 1. c. S. 117 .

9) 1. c. S. 78 . 
zellenartiges Gebilde entsteht, von den typischen mit andauernder SecretionsFähigkeit ausgestatteten Becherzellen trennen müssen. ......"

Mein Object Nummer 27 fixirte jch versuchsweise zunächst in verdünntem, dann erst in absolutem Alcohol und konnte nun das Oberflächenepithel, das an einzelnen Stellen den Oberendentypus deutlich zeigte, grösstentheils so verändert sehen, dass Vacuolenbildung, Aufbläbung in toto, Becherzellenformen, leere Zelltheken etc. und alle Uebergangsformen zwischen diesen, durcheinander gemischt, das vortäuschten, was ältere Autoren als totale Verschleimung bezeichneten. Dass es sich hierbei nur um ein Kunstproduct, respective um einen technischen Mangel handelt (Anwendung des verdünnten AIcohols) zeigen die ausführlichen Erörterungen ùber das Oberflächenepithel an meinen übrigen 30 Objecten.

In den Fällen von $\mathrm{L} u$ bar $\mathrm{sch}$ ist die Coincidenz zwischen Salzsäuremangel und dem Auftreten von Darmepithelschläuchen nicht auffallend, da er nur über Fälle mit Achylia gastrica spricht.

Die Befunde von $L$ e $\mathbf{k}^{1}$ ) zeigen nun, dass diese Coincidenz, die in Cohnheims Fällen so frappant hervortritt, keinen unmittelbar causalen Nexus bedeutet. Bei 9 von Leuk beschriebenen Fällen fehlt freie Salzsäure im Falle 5 und 9; sie wurde "einigemal" gefunden im Falle 7; in diesen 3 Fällen gab es weder Becher- noch Randsaumzellen; bei Fall 1, 4 und 8 dagegen, bei denen das Vorhandensein nicht unerheblicher Mengen freier Salzsäure ausdrücklich vermerkt ist, waren sowohl Becherzellen als auch Randsaumepithelien vorhanden. Es ist also hier nicht nur keine Coincidenz in obigem Sinne, sondern gerade das Gegentheil der Fall: Salzsäuregehalt und Darmepithelschläuche einerseits, Salzsäuremangel ohne Darmepithelschläuche anderseits.

Die Auffassung Leuk's bezüglich der Bedeutung dieser Gebilde ${ }^{2}$ ) ist eine vermittelnde: Dieselben Schläuche sollen, wenn sie spärlich vorkommen, als normal, wenn in grosser Menge, als pathologisch augesehen werden. Und zwar sollen sie normaliter auch im Fundus, allerdings in geringer Anzahl, vorkommen, wozu ich aber bemerken muss, dass letzterer Ausspruch ein wenig gewagt erscheint, da er auf einer einzigen Beobachtung (Fall 1) beruht.

Ich gehe nun an die Besprechung derjenigen unter meinen Präparaten, die Darmepithelschläuche aufwiesen.

Fall 3. Boczán Albert, 37 Jahre alt, klagt am 27./1. 1900 über Stuhlbeschwerden und morgendliches Erbrechen; Alcohol-Missbrauch zugestanden. Sehr gut genährt; Magen nuchtern stets leer; Ausheberung nach Probefrühstück ergab stets totalen Salzsäuremangel und reichliche Mengen ron

1) Leuk. Untersuchungen zur pathol. Anatomie des menschlichen Magens mit Berücksichtigung der practischen Verwerthbarkeit anatomisch diagnosticirter Magenschleimhautstückchen. - Zeitschrift für clinische Medicin. Band 37. 1899.

2) 1. c. S. 303 . 
Schleim. Diagnose: chronischer Magencatarrh. Golegentlich der fortgesetzten Untersuchungen mit dem Magenschlauch erlangte ich am 3./4., 25./4. und 8./5. 1900 je ein kleines Stückchen Magenschleimhaut.

Fall 4. Till Leopold, 45 Jahre alt, klagt am 5./12. 1899 über zunehmende Schlingbeschwerden; bei fortgesetzten Sondirungen stösst die Sonde constant, in einer Entfernung von $32 \mathrm{~cm}$ von den Schneidezähnen gerechnet, auf ein unüberwindliches Hinderniss. Wegen zunehmender Schwäche und da die ausgesprochene Cachexie die Diagnose: Speiseröhrenkrebs sichert, wird Anfangs Februar 1900 die Gastrostomie in zwei Sitzungen ausgeführt, bei der zweiten ein Stückchen hervorquellender Magenschleimhaut mit der Scheere abgetragen und der mikroskopischen Untersuchung zugeführt. Die Feststellung der chemischen Reaction im eröffneten Magen wurde leider unterlassen.

Fall 6. Frl. Gr . . . . ., 27 Jahre alt, klagt über verringerten Appetit. Druck und Völle nach dem Essen; Athembeklemmungen; dieser Zustand soll bereits zwei Jahre dauern. Zwei Stunden post mensam an der gut genähn ten Patientin ausgiebiges Plätschern in der Magengegend, das sich bis zwei Querfinger unter die Nabelhöhe erstreckt. Der nach dem Probefrühstück exprimirte Mageninhalt enthält $0,5 \%$ freie Salzsäure, keinen Schleim. Bei wiederholten Expressionen wechselnder Befund: Salzsäure bald fehlend, bald in sehr geringen Mengen. Bei einer Gelegenheit zwei kleine Schleimhautfetzen.

Fall 13. Vitányi (?) Jahre alt; seit einem Jahre magenleidend, häufiges Erbrechen saurer Massen. Stark abgemagert; Magen dilatirt; in Nabelhöhe ein grosser harter Tumor zu fühlen, der stagnirende Mageninhalt sowohl, als auch das exprimirte Probefrühstück weisen totalen Salzsäuremangel auf. Diagnose: Carcinoma pylori. Am 6./11. 1900 Gastoenterostomie; ein kleines Stückchen vorquellender Magenschleimhaut wird abgeschnitten und der mikroskopischen Untersuchung zugeführt.

Fall 23. Hecht Georg, 49 Jahre alt; grosser Tumor im Epigastrium rechts; Salzsäure fehlend; Gastroenterostomie am 12.2.1901; ein vorquellendes Schleimbautstückchen wird abgeschnitten und der mikroskopischen Untersuchung zugeführt.

In den Schnitten aus diesen fünf Objecten finden sich die von den Autoren beschriebenen:

a) Darmepithelschlauche und zwar konnte ich sie bei Fall 4, 13 und 23, wo sämmtliche Schnitte bis zur Muscularis erhalten waren, bis zum blinden Ende verfolgen; im Falle 3 und 6 dagegen, wo die unteren Schichten fehlten, nur auf eine kurze Strecke hin. Ihre Anzahl war eine sehr verschiedene: im Falle 3 und 4 beherrschten sie das Gesichtsfeld, kamen bei Fall 13 zu zwei bis vier gruppirt, bald in der Mitte bald an einer Seite der Schnitte vor; bei Fall 6 und 23 endlich fand ich nur ein bis zwei solcher Schläuche und zwar nur in einigen Schuitten aus diesen Objecten. -

Die Schläuche sind in ibrem ganzen Verlauf von nahezu gleichmässiger Dicke (Fig. 7, 8); von einer oberen Erweiterung, 
die dem Drüsenvorraume entsprăche, ist kaum etwas zu sehen. Die epitheliale Auskleidung besteht in einem einschichtigen Cylinderepithel ohne Oberende, das an gut gefärbten Prăparaten deutlichsten Randsaum zeigt. An dickeren Stellen des Schnittes ist an diesem Randsaum keine feinere Structur zu erkennen; an dünneren Stellen sieht man ihn bei stărksten Vergrösserungen bald deutlich gestreift (parallel der Längsachse der Zelle), bald sogar wie aufgefranst (Fig. 17). Diese Randsaumepithelien sind in oft ganz regelmässigen Abständen von Becherzellen unterbrochen, deren freies Ende offen erscheint. Bei HämatoxylinEosin-Färbung nehmen die Cylinderzellen rothe, die Becherzellen dagegen eine blassblaue Farbe an. Einen blassblauen Farbenton nimmt auch der im central gelegenen, engen Lumen des Schlauches angesammelte Schleim an. Viel anschaulichere Bilder liefert die Thioninfärbung in der von mir angegebenen Modification: gegen die durchwegs blau gefärbten Cylinderzellen stechen die violettrothen Becherzellen (Fig. 8, 9, 10) grell ab; da der im centralen Lumen der Schläuche enthaltene Schleim ebenfalls roth gefärbt ist und dieser Schleim mit dem noch in den Becherzellen befindlichen zusammenhängt, entsteht die abgebildete traubenförmige Zeichnung. Am Querschnitt der Schläuche sieht man (Fig. 10) genau das, was auch Cohnheim abgebildet hat.

An besonders dünnen Schnitten, die mit Thionin gefürbt wurden, zeigt der Randsaum bei sehr starker Vergrösserung eine vom Zellkörper verschiedene Fürbung; während diese nămlich in einem blaugrauen Farbenton erscheinen, sehen wir (Fig. 17) den Randsaum rein himmelblau gefarbt. Genau dasselbe Verhalten sehen wir auch an den Randsaumepithelien und Becherzellen des Dünndarmepithels (Fig. 11 und 19).

b) Das interglanduläre Gewebe, das breite Interstitien zwischen obigen Schläuchen bildet, enthält nur spärlich Bindegewebsfibrillen, in reichlichster Menge Lymphocyten, eine wechselnde Anzahl von Plasmazellen, spärlich Mastzellen, wenig Leucocyten. Ich konnte an diesem Gewebe ausser den Plasmazellen, deren Natur noch fraglich ist, nichts Entzündliches wahrnehmen: keine Stauung in den Capillaren, keine Erweiterung, Schlängelung oder Vermehrung derselben, auch keine Bindegewebs-Neubildung, sondern nur ein sehr zellenreiches, adenoides Gewebe, das an gewissen Stellen des Darms genau in derselben 
Form vorkommt, wie auch Ebnerl) ausdrücklich bemerkt: "Im Pförtnertheil des Magens geht das Bindegewebe der Schleimhaut in adenoides Gewebe über, von derselben Beschaffenheit wie im Darm."

Schnitte aus dem Magen Nr. 4 könnten nicht treffender abgebildet sein als in Figur 998 auf Seite 204 bei EbnerKo elliker, die sich doch auf die Schleimhaut des Wurmfortsatzes bezieht.

Im selben Sinne schreibt auch Bonnet $\left.{ }^{2}\right): \ldots \ldots \ldots$ viel reichlicher ...... ist . . . . . die Bindesubstanz im Pylorus, gleichzeitig ausgezeichnet durch eine sehr bedeutende diffuse Infiltration mit Leucocyten ......." Es ist nicht daran zu zweifeln, dass die grösste Zahl der von Bonnet als Leucocyten angenommenen Gebilde solche waren, die wir heute als Lymphocyten bezeichnen.

Die noch sehr fragwürdigen Plasmazellen, die von Manchen als Zeichen sehr gelinde verlaufender Entzündungsprocesse angesehen werden, können mit den Darmepithelschläuchen in gar keine Verbindung gebracht werden; denn einmal sind sie zwischen diesen nur in geringer Zahl anzutreffen, ein anderesmal wieder in grossen Mengen an Objecten, die gar keine Darmepithelschlauche aufweisen.

c) In dieses adenoide Gewebe eingebettet, finden sich bei Fall 4, 13 und 23 ausser den soeben beschriebenen Darmepithelschläuchen Längs- und Querschnitte von Drüsen, die einen ganz anderen Character aufweisen; sie haben meistens ein weites Lumen (Fig. 12), sind oft kolbig aufgetrieben, ihre EpithelialAuskleidung besteht aus niederen meist cubischen Zellen mit glashellem Inhalt und platt der Basis anliegendem Kern; mit einem Wort: unverkennbare Pylorusdrüsen ${ }^{3}$ ). Andere sind ganz ăhnlich gebaut, nur finden sich in der sonst ununterbrochenen Reihe der soeben beschriebenen kleineren Zellen weit grössere mit runden, mehr central gelegenem Kern. Diese färben sich bei Hämatoxylin-Eosin-Behandlung intensiv roth, während die übrigen blassroth bleiben; mit Thionin färben sich erstere hell-

1) 1. c. S. 168 .

2) B on net. Demonstrationen über den feineren Bau der Magenschleimhaut des Menschen etc. Deutsche medic. Wochenschr. 1893 No. 18.

3) Siehe bei 0 p pel l. c. S. 475 , Fig. 375. 
blau (Fig. 12) wie alle Belegzellen des Fundus im Gegensatze zu den übrigen hellrosenroth gebliebenen. Nach dem, was Stöh $\mathrm{r}^{1}$ ) und $O \mathrm{ppel}^{2}$ ) über die intermediäre Zone, das heisst Uebergang zwischen Pylorus und Fundusschleimhaut, schreiben und abbilden, kann es gar keinem Zweifel unterliegen, dass wir es hier mit Pylorusdrüsen zu thun haben, die aber die Nähe des Fundus durch das mehr oder minder sporadische Auftreten von Belegzellen erkennen lassen.

d) $\mathrm{Zu}$ unterst gegen die Muscularis zu finden sich in jedem Schnitte aus Object 4, 13 und 23 solitäre Follikel; die grosse Zahl derselben zeigt ebenfalls die pylorische Gegend oder wenigstens deren Nähe $a{ }^{3}{ }^{3}$ )

Deradenoide Characterdesinterglandulären Gewebes, die Anwesenheit von Drüsenformen, die der Pylorusgegend oder der intermediären Zone angehören, die zahlreichen solitären Follikel lassen gar keinen Zweifel daran, dass die Schleimhatpartikelchen den genannten Magenregionen angehören und, abgesehen von den Darmepithelschlauchen und vielleicht auch von den Plasmazellen, in gar keiner Hinsicht von normalen, jene Gegenden characterisirenden Gewebe sich unterscheiden.

Es ergiebt sich nun die Frage, welche Rolle den Darmepithelschläuchen in dieser als pylorisch (oder intermediär) erkannten Magenregion zukommt?

1. Zunächst soll festgestellt werden, dass sie auch im normalen Magen vorkommen. Schmidt ) fand sie "nur in einem Magen (Nr. 4) ......, in dem auch jenseits der Grenze im Magen selbst noch einzelne versprengte Darmepithelien angetroffen wurden ...... Auch $\mathrm{Lubarsch}$, der die Darmepithelschläuche als pathologische Gebilde ansieht, hat sie nur einmal im normalen Magen gesehen und zwar ${ }^{5}$ ) bei einem 22 jährigen Hingerichteten, „bei dem dicht am Pylorusring die

1) Siehe $O$ p pel' sches Excerpt 1. c. S. 473.

2) 1. c. S. 469 .

3) E bner l. c. S. 169 .

4) 1. c. 1896 . S. 487 .

5) 1. c. S. 132 . 
Magenschleimhaut geradezu den Character der Duodenalschleimhaut angenommen hatte."

Weit wichtiger ist für unsere Betrachtung, dass $\mathrm{S} \mathrm{ch}$ a f f e $\mathrm{r}^{1}$ ) und $\mathrm{Ebner}^{2}$ ), die doch über normale Histologie arbeiteten, die in einer Entfernung von $10-12 \mathrm{~mm}$, resp. $1-2 \mathrm{~cm}$ vom Pylorus vorkommende Darmepithelschläuche als normale Vorkomm is se beschreiben.

Wir hätten also festgestellt, dass das Vorkommen der Darmepithelschlăuche in der Nähe des Pförtners durchaus nichts pathologisches an sich hat. Nun wurden aber meine Objecte 4, 13 und 23 durch Gastrostomie resp. Gastroenterostomie gewonnen. Die technischen Umstände dieser Operationen bringen es mit sich, dass der Magen nicht hart am Pylorus, sondern immer in einiger Entfernung von demselben eröffnet wird; daher die Schleimhautstückchen wohl aus der $\mathrm{N}$ ähe des Pylorus, doch nicht aus seiner unmittelbaren Nachbarschaft herstammen können. Es ergiebt sich also die zweite Frage:

2. Ob unter Umständen auch eine vom Pylorus in grösserer Entfernung gelegene Region der Magenschleimhaut pylorischen (oder intermediären) Character haben könne? Wenn dies der Fall ist, so ist auch das Vorkommen von Darmepithelschläuchen in grösserer Entfernung vom Pylorus erklärt. Die Beantwortung dieser Frage fällt nicht schwer: Bereits $K u p f f e r^{3}$ ) fand die Ausdehnung der Pyloruszone einmal $6 \mathrm{~cm}$, die der Uebergangszone im selben Fall $3-4 \mathrm{~cm}$; in einem weiteren Falle bei einem Hingerichteten ${ }^{4}$ ) war „die Pylorus- und Uebergangszone ..... bis reichlich zur Mitte des Magens reichend, $14 \mathrm{~cm}$ von der Valvula Pylori entfernt, traf man noch immer den Bau dieser Region: tiefe, bis zur Mitte der Schleimhaut reichende Magengruben, kurze Drüsen mit vereinzelten Belegzellen."

Um wieder auf die normale Histologie mich zu berufen, führe ich $\mathrm{Ebner}^{5}$ ) an, der „die Ausdehnung des mit Pylorusdrüsen bedeckten Magenabschnittes ...... individuell sehr verschieden" fand.
1) 1. c. S. 90 .
2) l. c. S. 163 und 164 .
3) 1. c. S. 11 .
) 1. c. S. 19 .
5) 1. c. S. 163 . 
Solche individuelle Schwankungen zeigen sich auch an der Beschaffenheit der durch Gastrostomie und Gastroenterostomie gewonnenen Stückchen, wobei allerdings auch die Wahl der Operationsstelle am Magen eine gewichtige Rolle spielt. Eine ganze Anzahl zeigt unverkennbar pylorisches, andere wieder typisches Fundus-Gewebe; zuweilen findet man sogar intermediären Character sehr schön ausgeprăgt.

Wenn ich nach dem Obengesagten nun annehmen darf und ich habe allen Grund zu dieser Annahme - dass in den Fällen 4, 13 und 23 der Pylorusregion eine grössere Ausbreitung als in den meisten anderen Fällen hatte, wird auch das Auftreten der Darmepithelschläuche nichts Auffallendes mehr an sich haben: sie kommen für gewöhnlichnur in der Nähedes Pylorus vor, weil die pylorische Region eine eng beschrankte ist; hat diese letztere einoder das anderemal eine grössere Ausbreitung, so werden eben auch die Darmepithelschláche in grösserer Entfernung angetroffen werden. Von zehn unter ähnlichen Umständen operirten Făllen traf ich sie drei Mal an, während sie sieben Mal fehlten. -

3. Welche Momente sind es nun, die an den Darmepithelschlăuchen selbst erkennen lassen, dass sie keine pathologischen Gebilde sein können?

An den in Fig. 7 abgebildeten, mit Thionin gefärbten Schnitten aus Object 13 sieht man normale Drüsenvorräume, die das Gesichtsfeld rechts und links einnehmen, in der Mitte durch eine Gruppe von Darmepithelschläuchen unterbrochen; in ersteren sieht man, entsprechend den Oberenden, gleichmässige Rothfärbung aller Epithelien, in der letzteren dagegen eine Rothfärbung nur der in regelmässigen Abständen sitzenden Becherzellen, während die dazwischen liegenden Cylinderzellen blau blieben. Nach der von den Autoren vielfach vertretenen Anschauung wären diese Schlänche durch krankhafte Veränderung, speciell schleimige Degeneration, aus den normalen Drüsenvorräumen entstanden.

Kannmansichnun einen pathologischen Process so ablaufen denken, dass eine ganze Reihe von Zellen, deren jede einzelne früher ein schleimhaltiges Oberende besass, sich so verandere, dass der schleimgehalt nurmehr in einzelnen Zellen bestehen bleibt 
(diesmal den ganzen Zellleib einnehmend: Becherzellen), in allen anderen Zellen dagegen, die noch zudem einen Randsaum erhalten sollen, gänzlich schwinde?!

Einen weiteren Beweis gegen die Auffassung der Darmepithelschlanche als pathologische Gebilde liefert auch Fig. 18, die nicht nur die scharfe Grenze zwischen beiden Epithelarten zeigt, so dass von einem Uebergang nichts zu sehen ist, sondern auch eine förmliche Stufe dort, wo die letze Randsaumzelle an die erste, ein Oberende besitzende Zelle stösst. Nach diesem ersten, am Object 13 erhobenen Befund liess ich es mir angelegen sein, die Grenze zwischen beiden Epithelarten in allen Fallen zu untersuchen, wo diese Schläuche vorkamen und fand überall dasselbe Verhalten. Selbstverständlich können es nur ganz dünne Stellen des Präparates sein, die für diese Untersuchung sich eignen.

Diese scharfe Grenze wird auch von mehreren jener Autoren angegeben, die trotzdem für den pathologischen Charakter der Schläuche eintreten.

Dieser Mangel an Uebergangsformen, die Stufenbildung, die die Grenze noch schroffer anzeigt, lassen keinen $Z$ weifel daran aufkommen, dass es sich um keine pathologisch veranderte MagenschleimhautElemente handelt, sondernum Gebilde, die eigentlich dem Darm angehören und hier als ganz fremdartige Elemente der Magenschleimhaut eingepflanzt sind.

4) Um das inselförmige Auftreten von Darmepithelschläuchen mitten zwischen normalen Drüsenvorräumen zu erklären, muss man daran denken, was Schmidt bereits $1896^{1}$ ) über versprengte Darmepithelien in der Pylorusregion vermuthete: "Sie bilden ein Analogon zu dem Capitel der "versprengten Drüsen“, für welches ebenfalls die Magenschleimhaut häufige Beispiele liefert. Am Bekanntesten ist in dieser Beziehung die als Uebergangszone bezeichnete Gegend zwischen Fundus und Pylorusschleimhaut, in welcher Fundus- und Pylorusdrüsen bunt durcheinander gemischt zu sein pflegen."

An einem Objecte Nr. 15, wo eie Darmepithelschlăuche nur in nächster Nähe des Pylorus vorkamen, und das ich deshalb

1) 1. c. S. 487 .

Archiv f. mikrosk. Anst. Bd. 58. 
nicht als sechstes zu den oberwähnten fünf Fällen nehmen wollte, war dieses Durcheinandergemischtsein der zwei heterogenen Elemente in ausgezeichneter Weise zu sehen. Das Uebergangsstück zwischen Magen und Duodenum (Frau Uitz, grosses Carcinom, Totalexstirpation des Magens) hatte ich in toto in Schnitte zerlegt, die folgendes Bild zeigten: Genau wie bei Fall 13 waren auch hier mitten in das Magengewebe mit normal geformten Drüsenvorräumen Darmepithelschläuche eingpflanzt, immer mit scharfer Abgrenzung der beiden Epithelarten. Weiterhin fanden sich aber auch Bilder, die ein Analogon zur Einmündung von Brunner'schen Drüsen in Lieberkühn'sche Drüsell bilden ${ }^{1}$ ). Es trat nämlich in den Vorräumen einzelner Pylorusdrüsenschläuche typisches Darmepithel an Stelle der gleichmässigen Cylinderzellen mit Oberenden; auch hier zeigten sich die beiden Epithelarten gegeneinander schroff abgegrenzt. Dieses Durcheinandergewürfeltsein heterogener Elemente ging aber noch weiter: Es fanden sich Drüsenvorräume, die nur an einer Wand und nur auf eine kurze Strecke hin Darmepithelien trugen und umgekehrt Darmepithelschläuche mit einer kurzen Reihe von Oberenden tragendem Magenepithel; auch hier schärfste $\mathrm{Ab}$ grenzung zwischen beiden Epithelarten.

Dieses Ineinandergreifen verschiedener Elemente, das zwischen Fundus- und Pylorusdrüsen schon lange bekannt ist und das soeben für Magen- und Darmelemente beschrieben wurde, findet sein Analogon darin, was Schaffer ${ }^{2}$ ) über Magen und Oesophagus schreibt: Sch affer sah an menschlichen Mägen, dass das Pflasterepithel $^{3}$ ) an der Grenze gegen den Magen grössere rundliche und unregelmässige Bezirke von Magenepithelien ganz umschliesst, so dass es durch diese Inselchen von Magenschleimhaut gitterartig durchbrochen erscheint.

Das inselförmige Auftreten von Darmepithelschläuchen in pylorischem Gewebe ist genau so aufzufassen.

5) Dass sie nicht an jedem Magen in gleicher Zahl und im selben Ausmaass vorkommen, ist einfach ndividuellen Schwankungen zuzuschreiben, die an anderen Stellen des Digestionstractus ebenfalls vor-

) s. Ebner. 1. c. S. 196. Fig. 991.

2) 1. c. S. 75 .

$\left.{ }^{8}\right)$ des Oesoghagus. 
kommen. Solche beschreibt Schaffer ${ }^{1}$ ) sogar betreffs der normalen Oesophagusdrüsen; weit grössere aber ${ }^{2}$ ) betreffs der von ihmsoge nannten oberen und unteren cordialen Oesophagusdrüsen, die auch typisches Darmepithel enthalten können. Er fand die ersteren (oberen) an 7 von 10 untersuchten Fällen in ungemein wechselnder Lage: von der Höhe des Ringknorpels bis zur Höhe des vierten bis fünften Luftröhrenknorpels; desgleichen in wechselnder Grösse, indem die Durchmesser 4 und $6^{1} / 2 \mathrm{~mm}$ in einem, 0,65 und $0,8 \mathrm{~mm}$ im anderen Falle betrugen. Ebenso verschieden fand er auch ihr mikroskopisches Verhalten.

Es ist also nichts Merkwürdiges, dass auch die Darmepithelschläuche im Magen, als versprengte Keime so grosse individuelle Verschiedenheiten aufweisen.

Letztere sucht Schaffer $r^{3}$ ) folgendermassen zu erklären: „Wenn man bedenkt, ..... dass bei der ontogenetischen Entwicklung der höheren Thiere das Magendarmrohr ursprünglich einen einheitlichen Epithelüberzug besitzt, dann erscheint die Erklärung des Vorkommens von darmdrüsenähnlichen Bildungen am Beginn und am Ende des Magens einfach darin zu liegen, dass es sich um, bei der secundären Differenzirung der typischen Magenschleimhaut stehen gebliebene Inseln von Darmschleimhaut handelt . . . . . . .

Bezüglich der grossen Schwankungen in der Vertheilung der versprengten Keime wäre nach $\mathrm{Schaffer}$ ) an "typische Unterschiede" zwischen grosser und kleiner Curvatur, zwischen vorderer und hinterer Magenfläche zu denken. Diesbezügliche eingehende Untersuchungen sind noch der Zukunft vorbehalten, wobei aber nicht $\mathrm{zu}$ verhehlen ist, dass ihrer Ausführung grosse technische Schwierigkeiten, namentlich in der Beschaffung des Materials im Wege stehen.

Ich sagte Seite 706 dass die von mir untersuchten Schleimhautpartikelchen der Pylorusregion oder intermediăren Zone angehören und "abgesehen von den Darm-
1) 1. c. S. 60 .
2) 1. c. S. $66 \mathrm{ff}$.
s) 1. c. S. 91.
4) 1. c, S. 92. 
epithelschläuchen und vielleicht auch den Plasmapellen in gar keiner Hinsicht von normalem, jene Gegenden characterisirendem Gewebe sich unterscheiden." Ich darf nun wohl hinzufügen: a uch die Darmepithelschläuche sind keine pathologische Gebilde sondern durchaus normale Vorkommnisse, deren Zahl und Ausbreitung abersehr grossenindividuellen Schwankungenunterliegt.

Da ich mich auf diese Weise in strictem Widerspruch zu der Ansicht der meisten Autoren befinde, die die Darmepithelschläuche im menschlichen Magen als pathologische Bildungen bezeichnen, will ich nun an der Hand der betreffenden Publicationen versuchen, ihre Befunde mit meiner Auffassung in Einklang zu bringen. Ich muss soweit wie irgend möglich nachweisen - und dieser Nachweis ist selbstverständlich nicht immer leicht - dass die von den Autoren untersuchten Schleimhautstückchen in der Regel aus der Nähe des Pylorus herstammen. Als einen wichtigen Beleg zur Unterstützung meiner obigen Auslassungen werde ich auch ihre Angaben über die inselförmige Anordnung der Darmepithelschläuche so wie über die scharfe Grenze zwischen beiden Epithelarten in erster Linie berücksichtigen müssen.

Bezüglich der Bedenken, die die von Kupfer ${ }^{1}$ ) im Magen beobachteten Becherzellen erwecken müssen, verweise ich auf das Seite 698 Gesagte.

G. M e y e ${ }^{2}$ ) beschrieb die Darmepithelschläuche in zwei Fällen und gab auch treffende Iltustrationen zu denselben. In einem Falle handelte es sich um eine Atrophie der Magenschleimhant, welche Darmepithelschlänche „nur in der Pylorusgegend" zeigte; den zweiten ähnlichen Befund erhob or an einem ,durch Resection wegen Pyloruscarcinom gewonnenen und lebenswarm in Alcohol gebrachten Stück von Magenschleimbaut."

$\mathrm{Sachs}^{3}$ ) fand die von ihm sog. "Magenschleimdrüsen" bei folgenden 7 von 13 daraufhin untersuchten Fällen: Fall A. starb an Kaiserschnitt (indicirt durch Eclampsie); C. und D. starben an Pneumonie; F. litt an Magengeschwür und Pleuritis; G. an Phthise; H. an Phthisis pulmonum et laryngis; $N$. war lange leidend und starb an Metastasenbildnng nach einer

1) $1 . \mathrm{c}$.

2) $1 \mathrm{c}$.

3) 1. c. 
traumatischen Panophthalmitis. Es sollen demnach eine ganze Reihe von Erkrankungen, in der Regel von Infectionskrankheiten die Fäbigkeit besitzen, eine so abenteuerliche Veränderung des Magens zu bewirken.! - Man könnte dies noch - mit viel gutem Willen - bei Fall F., G., H. und N. zugeben, wo es sich offenbar um ein langes Siechthum handelt; doch keinesfalls bei Fall A., C. und D., wo acute Processe in relativ kurzer Zeit zum Tode führten. - Spätere Autoren liessen die Darmepithelschläuche bei Krankheitsprocessen entstehen, die im Magen selbst abliefen: das hätte noch den Anschein die Plausibilität; dass aber Erkrankungen entfernter Organe, dazu auch acute Processe, die Umwandlung des Magenepithels in Darmepithel bewirken sollen, halte ich für eine Auffassung, die nicht erst bekämpft werden muss.

Waren daher die eigenartigen Drüsen, die $S$ a $\mathrm{c} h \mathrm{~s}$ bei 7 von 13 Fällen (nicht Magenkrankheiten !) anführt, richtig die in Frage stehenden Darmepithelschläuche, so können sie nicht als pathologische Bildungen aufgefasst werden, sondern nur als durch individuelle Schwankungen bedingte Vorkommnisse.

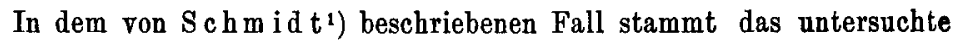
Schleimhautstückchen aus der Pylorusregion, wie dies in der Beschreibung ausführlich bemerkt ist. Er beschreibt das intergranduläre Gewebe in diesem Fall als pathologisch infiltrirt und stützt sich dabei auf die irrthümliche Annahme, dass " . . . . . . . . normalerweise in der Pylorusgegend das Bindegewebe gegen die zelligen Elemente völlig zurïcktritt." Nacb dem, was ich über diese Verbältnisse weiter oben mittheilte, halte ich eine weitere Erörterung für überflüssig.

Schmidt ist der Meinung, dass, ,derartige Epithelschläuche auch unter normalen Verhältnissen vereinzelt zwischen den Labrrüsen, ganz besonders der Pylorusgegend, angetroffen werden..... ." Das von $\mathrm{Sc}$ hmidt 1895 beschriebene Schleimhautstückchen fällt demnach unter dieselbe Beurtheilung wie meine Objecte 4, 13 und 23: es stammt aus der Pylorusgegend und hat nichts Pathologisches an sich.

Bei Cohnheim's Fällen sind vielfach unverkennbare Merkmale pylorischen Gewebscharacters angeführt; so beschreibt $\mathrm{er}^{2}$ ) bei Fall 19, wo Becherzellen in ungeheurer Anzahl vorkamen, in der tiefen Schicht.... in stark erweiterten, keulenförmig am Ende aufgetriebenen Drüsen grosse B.-Z. ${ }^{3}$ ) aber nur sporadisch . . .' . . sind fein gekörnt .. . . . .“ Diese Beschreibung passt vortrefflich anf die (Figur 12) abgebildeten Drüsen aus der Uebergangszone: Die spärlichen Belegzellen zeigen die Nähe des Fuudus an, die weiten Lumina aber die der Pars pylorica. Dass „Becherzellen . . . . . niemals in der Nähe der B.-Z. $\left.{ }^{8}\right)^{4}$ sich finden, rührt solbstverständlich davon her, dass letztere, also Belegzellen, nur in Drüsen vom Magentypus vorkommen können, nie aber in solchen vom Darmtypus,

1) 1. c. 1895 .

2) l. c. S. 286 .

s) Abkürzung für Belegzellen. 
d. i. in Darmepithelschläuchen. - Bei Cohnheim's Fall 25 deuten die zahlreichen solitären Follikel auf die pylorische Provenienz des Stückes.

Das von mir mehrfach erwähnte inselförmige Auftreten von Darmepithelschläuchen mitten zwischen Drüsenvorräumen ist auch schon von Cohnhe i m beobachtet, nur anders interpretirt worden. So beschreibt er zum Beispiel an Flachschnitten aus Fall 17 „Querschnitte von Vorräumen mit Becherzellen und Stäbchensaumepithel, neben Querschnitten von einfachen, schleimige Cylinderepithelien tragenden Vorräumen . . . Diese gruppenweis abwechselnde Anordnung von Darmepithel- und Cylinderzellenschläuchen fand er an 7 von den 10 Schleimhautstückchen, die Darmepithelschläuche trugen $(17,18,19,21,27,29,30)$.

So schwer die Beweisführung auch ist, glaube ich wenigstens für einzelne der $\mathrm{Coh} \mathbf{n} \mathrm{h}$ e i m 'schen Objecte die Abstammung aus der pylorischen oder intermediären Region, für die meisten aber die inselförmig versprengte Anordnung der Darmepithelschläuche erwiesen zu haben, daher diese Co h nhe i m'schen Befunde in morphologischer Hinsicht meiner Annahme nicht widersprechend sind.

Ich will auch nicht unerwähnt lassen, dass speciell $\mathrm{Coh}$ n beim's Angaben über entzündliche Processe oder Zeichen der Atrophie, wie Vermehrung der Capillaren des interstitiellen Bindegewebes, die Durchwanderung von Leucocyten, die degenerativen Veränderungen an Haupt- und Belegzellen mit meiner Auffassung der Darmepithelschläuche, als nicht pathologische Gebilde, durchaus nicht in Widerspruch stehen. In meinen hierher gehörenden Fällen habe ich wohl ähuliche Veränderungen nicht gesehen; selbstverständlich können sie in anderen Fällen in ausgedehntestem Maasse vorkommen; nur baben die Darmepithelschläuche mit diesen Processen nichts zu thun. Diese können eben Schleimhatregionen, die Darmepithelschläuche aufweisen, ebensobefallen, wie solche, in denensiefehlen.

$\mathrm{S} \mathrm{chmid} \mathrm{t} \mathrm{bezeichnet}{ }^{1}$ ) in seiner zweiten Publication vom Jahre 1896 als .... Hauptfundort der Darmepithelschläuche .... die Pylorusschleimhaut. Wenn sie spärlich vorhanden sind, sind sie eigentlich nur hier anzutreffen." Da Sch midt die fraglichen Gebilde in dieser Publication als pathologische Bildungen auffasst, legt er besonders Gewicht auf vermeintliche Zeichen eines pathologischen Processes; so erwähnt er auch ${ }^{2}$ ) „Anhäufung von Follikeln", was aber einfach davon herrührt, dass die Schnitte aus der Pylorusregion stammen.

Auch die scharfe Grenze zwischen Magen- und Darmepithel, die ich als einen der stärksten Belege zu meiner Auffassung ansehen muss, betont er wiederholt ${ }^{3}$ ).

Am Rand und in der Umgebung heilende Magengeschwüre hat $\mathrm{Schmidt}{ }^{4}$ ) in drei Fällen nach Darmepithelschläuchen gesucht. Er fand sie nicht im Falle 17, wo das Geschwür an der grossen Curvatur sich fand,

1) 1. c S. 496 .

2) 1. c. S. 495 .

$\left.{ }^{3}\right)$ 1. c. S. 486 und 495 .

4) l. c. S. 503 . 
wohl aber im Falle 16, wo ,an der Uebergangsstelle des Fundus in die Pylorusschleimhant ein Zehnpfennigstück grosses Ulcus mit starken Rändern ${ }^{\star}$ sass Also befanden die Darmepithelschläuche sich $z$ weifellos wieder in der verbreiterten regio pylorica oder intermedia. Fall 15 verhielt sich mikroskopisch ebenso; doch erwähnt Shmidt nicht, ob die an der kleinen Curvatur sitzenden Geschwüre nahe zum Pylorus oder weit von ihm entfernt waren. Dieser letztere Fall ist daher nach keiner Richtung hin beweisend.

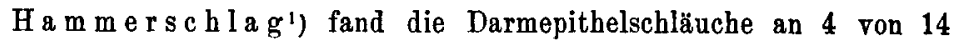
darauf untersuchten Stückchen; und zwar erhielt er dieselben dreimal bei Resectio pylori, neunmal gelegentlich der ausgeführten Gastroenterostomie und zweimal ex cadavere. Zwei Fälle (10 und 13) von den drei durch Resectio pylori gewonnenen Stückchen, die also zweifellos aus der Nähe des Pylorus herstammten, trugen Darmepithelschläuche, desgleichen 2 (Fall 4 und 9) von den 9 durch Gastroenterostomie gewonnenen Stückchen, während keine der beiden aus dem Fundus der Cadavermagen herausgeschnittenen Stücke diese Schlüuche zeigten. Nun giebt $\mathrm{Hammerschlag}{ }^{2}$ ) an, dass die 9 bei Gastroenterostomie gewonnenen Stückchen "durchweg dem Fundus" angehörten. Diesbezüglich glaube ich bemerken zu müssen, dass einerseits die verschiedenen Methoden dieser Operation, wie G. anterior, G, posterior etc. grosse Verschiedenheiten in der Wahl der anzuschneidenden Magenregion aufweisen - wie dies in den chirurgischen Handbüchern ${ }^{3}$ ) zu ersehen ist -, dass aber anch andererseits derselbe Operateur, wenn er stets nach derselben Methode operirt, durch verschiedene Umstände, wie Lage und Grösse der Strictur, beziehungsweise des Tumors, durch etwaige Formveränderung des Magens im Sinne einer Ectasie gezwungen wird, dem Pylorus bald näher zu kommen, bald sich wesentlich von ihm zu entfernen.

Meine 8 durch Gastroenterostomie gewonnenen Objecte gehörten, mit Ausnahme von zweien, solchen Patienten an, die vom selben Operateur, Docent Herczel, operirt wurden; und zwar wurde jedesmal eine G. posterior ausgeführt. Und doch zeigte ein Theil dieser Schleimhautstückehen (6) typischen Fundus, ein anderer Theil (2) typisches Pylorusgewebe. Aus diesem Grunde halte ich es nicht für wahrscheinlich, dass alle durch Gastroenterostomie gewonnenen Stucke $\mathrm{Hammerschlag's} \mathrm{dem} \mathrm{Fundus}$ angehörten; vielleicht waren gerade die Stücke 4 und 9 solche, die aus der Nähe des Pylorus herstammten. Ich kann dies selbstverständlich nicht beweisen; immerhin ist es aber auffallend und für meine Ansicht sprechend, dass von 9 vermeintlich und 2 sicher aus dem Fundus stammenden Stücken nur zwei Darmepithelschläuche trugen. Im Uebrigen sagt Ha m mer-

1) 1. c. 1896 .

9) 1. c. 1896, S. 200 .

3) Siehe Illustrationen bei Esmarch-Kow alzig. Chirurgische Technik. III. Auflage. 3. Band 1899; Berg man n-Bru s - M i k u licz. Handbuch der practischen Chirurgie; bei Forgue-Reclus, Traité de therapeutique chirurgicale heisst es Seite 739 sogar ausdrücklich: On choisira donc le fond de la poche prepylorique, trés prés de la grande courbure. 
schlag selbst'), dass man die fraglichen Schläuche "besonders bäufig in den regio pylorica, seltener in den Schleimhautstücken vom Fundus" findet.

Lubarsch ${ }^{2}$ ) standen von 11 Kranken 20 durch den Magenschlauch losgerissene Schleimhautstücke zur Verfügung, von welchen 6 Stücke Becherzellen und Randsaumepithelien trugen: von Fall 6 Stücke 2 und 3 ; von Fall 7 Stücke 1 und 2; von Fall 8 Stücke 2 und 3. In der Lubarschschen ausführlichen Beschreibung vermissen wir bei keinem einzigen di eser 6 St ü cke Merkmale pylorischer oder intermediärer Provenienz: so sind bei einem Stückchen ausdrücklich „Drüsen vom Character der Pylorusdrüsen" verzeichnet; weiterhin sind es bald S t $00 \mathrm{~h} \mathbf{r}$ 'sche bald wieder $\mathrm{Nu}$ ssb a $u$ m 'sche Zellen, die bekanntlich ${ }^{3}$ ) beide nur in der Pylorusregion vorkommen.

Sollten die letztgenannten, wie $\mathrm{L} u$ b a $\mathrm{r} s \mathrm{c}$ vermuthet, nicht $\mathrm{Nuss-}$ b a $\mathrm{u}$ 'sche sondern $\mathrm{Pa} n \mathrm{eth}$ 'sche Zellen sein, so haben sie fïr die $\mathrm{Be}$ stimmung der Magenregion dieselbe Bedeutung, denn ihr gewöhnlicher Fundort ist der Darm; auf den Pylorus und auf die regio pylorica seben wir sio nur (wie die Darmepithelschläuche) in vereinzelte n Exemplaren übergreifen.

Es resultirt demnach aus Obigem, dass auch $L$ a barsch nur an solchen Schleimhantstückchen Darmepithelschläuche sah, die aus der regio pylorica oder intermedia herstammten.

Wie wenig Plausibilität der Erklärung der Darmepithelschläuche als pathologisch veränderte Drüsenvorräume innewohnt, geht am klarsten aus I $\mathrm{u}$ b a rsch's folgender Beschreibung derselben hervor:,$\ldots \ldots \ldots$ die Aehnlichkeit der veränderten Magenschleimbaut mit Darmschleimhaut ist so gross, dass man bei unseren Befunden in Fall 6 und 7 - wenn es nicht a priori ausgeschlossen wäre und zudem durch den Befund von Labdrüsen völlig unmöglich gemacht würde - versucht sein könnte, zu erörtern, ob man es nicht mit Darmschleimhaut zu thun habe. Bis in die kleinsten Einzelheiten ist diese Uebereinstimmung da: das Auftreten der Becherzellen, die Durchwanderung von Leucocyten, die Massenhaftigkeit der Mitosen, die grosse Zahl von acidophilen Wanderzellen und endlich das Vorkommen der fuchsinophilen Körnchen machen diese Uebereinstimmung zu einer vollkommenen."

Je mehr diese Uebereinstimmung die Prüfung auf ihre Richtigkeit besteht - und Lubarsch prüfte, wie ans seiner Arbeit ersichtlich, mit äusserster Rigorosität, - um so eher scheint es mir ausgeschlossen, dass es sich um einen pathologischen Process handeln könne.

Lubarsch führt, wie früher auch Cohnheim, je einen Fall an, wo das von demselben Kranken zu einem späteren Zeitpunkte gewonnene Schleimhantstückchen bereits Bildung von Darmepithelschläuchen aufwies im Gegensatze zu dem früher erhaltenen, das normales Oberflächenepithel trug. Dem kann ich meinen genannten Fall 3 entgegenstellen, von dem ich am 3. 4. ein Schleimhautsückchen erhielt, das typische Darmepithelschläuche

1) 1. c. S. 206.

8) l. c.

8) Oppel l. c. S. 246 . 
aufwies, am 25. 4. und 8. 5. desselben Jahres, also jedenfalls um Wochen später, nur Stückchen mit vollständig normalem typischem Magenepithel.

Wird daher in Lubarsch's und Cohnheim's Fällen ein progredienter Krankheitsprocess angenommen, der Darmepithelschläuche zeitigt, so müsste ich bei meinem soeben citirten Fall eine regressive, eigentlich regenerative Umwandlung von Darmepithel in Magenepithel annehmen. - Davon kann aber selbstverständlich keine Rede sein; um so weniger, da ja der chronische Catarrh während der ganzen Beobachtungszeit unverändert blieb.

Es liegt viel näher, so zusagen auf der Hand, anzunehmen, dass zufälligerweise bei Lubarsch und Cohnheim das später -, bei meinem Falle aber das zuerst gewonnene Schleimhautstückchen von derjenigen Magenregion losgerissen wurde, welche eben Darmepithelschläuche trug.

Leu k sah ${ }^{1}$ ) die Darmepithelschläuche dreimal, bei Fall 1,4 und 8 ; und zwar enthielt das betreffende Magensecret, wie bereits oben erwähnt, jedesmal mehr minder ansehnliche Mengen freier Salzsäure. Bei Fall 4 und 8 ist wieder ausdrücklich erwänht, dass die Darmepithelschläuche in der Umgebung des Pylorus, bei Fall 1 dagegen, dass sie in der dem Fundus zu gelegenen Umgebung des resecirten Tumors lagen.

Dieser letztere wäre der einzige einwandfreie $\mathrm{Fall}^{2}$ ), wo Darmepithelschläuche am Fundus vorkamen.

Bei der Analyse dieses Befundes stellt $\operatorname{sich}^{3}$ ) nun Folgendes heraus : Laut Leuk's Beschreibung ist am betreffenden Objecte " . . . . . das Bindegewebe schmal und die Epithelschläuche bis zum Grunde gerade und parallel . . . . . , der gauze Process macht denselben nicht entzündlichen Eindruck, den man bei Betrachtung der „einfachen Schleimdrüsen“ Kupffer's erhält. . . . . . . " Auf diese Betonung des Mangels an Entzündungserscheinungen lege ich grosses Gewicht, da ich hieraus wieder nur ersehen kann, dass die Darmepithelschläuche auch in diesem Falle keine pathologische Bildungen sein können. Wenn Le uk an diesen Gebilden trotz seines soeben citirten Eindruckes ,einen von Grübchenepithel ausgehenden Regenerationsversuch des durch Atrophie zu Grunde gehenden Drüsenkörpers" sieht, macht er diese Concession gewiss nur der auch von ihm angenommenen Doctrin, die die Darmepithelschläuche als pathologische Ge bilde erklärt.

Spricht dieses ganz vereinzelte Vorkommen von Darmepithelschläuchen am Fundns gegen meine Annahme, dass dieselben nur versprengte Darmelemente seien? Ich glaube nicht! Die grossen individuellen Sehwankungen, die - wir wir gesehen haben - bezüglich versprengter Epithelien auch sonst am Digestionstract vorkommen, machen auch den Le $\mathfrak{k}$ 'schen Fall Nr. 8 erklärlich. Wenn typische Darmepithelien nicht nur an der Cardia und in den sog. unteren, sondern auch in den sog. oberen cardialen

1) $1 . \mathrm{c}$.

2) Der Seite 715 besprochene Fall Nr. $15 \mathrm{Sc} \mathrm{hm}$ idt's, bei dem die Ulcera an der kleinen Curvatur sassen, könnte obigem Falle angereibt werden, wenn bei der wenig präcisen Ortsbezeichnung die Nähe des Pylorus auszuschliessen wäre.

s) 1. c. S. 309 . 
Oesophagusdrüsen (Sch affer) in der Höhe der ersten Luftröhenkn orpel vorkommen, so ist es ganz gut denkbar, dass Darmepithelschläuche ausser an ihrem häufigen Fundorte, der regio pylorica und intermedia, anch ein oder das anderemal am Fundus zu finden sein werden.

Die vielfach constatirte Coincidenz zwischen Salzsăuremangel und dem Auftreten der Darmepithelschläuche ist wohl geeignet, einen Causal-Nexus annehmen zu lassen, da ja Salzsăuremangel im Secret eine recht characteristische Eigenschaft des kranken Magens darstellt.

Boas, der überbaupt als Erster auf die grosse Bedeutung der durch den Magenschlauch losgerissenen Schleimbautstückchen hinwies und dieselben eingehend untersuchte, sagt bereits 1894'1): „Besonders häufig kommt es zu Schleimhautexfoliationen in Fällen von chronischer Gastritis . . . . . ; ich habe hierbei den Eindruck gewonnen, als ob manche Schleimhäute stark aufgelockert sind und daher schon ein geringer Insult . . . . . kleine Ablösungen der Mucosa hervorbringen kann." Aus dem Umstande, dass die chronische Gastritis sehr häufig mit Salzsäuremangel einhergeht, folgt unmittelbar, dass Schleimhautstückchen besonders häufig aus solchen salzsäurelosen Mägen erhalten werden.

Dasselbe sagt auch $\mathrm{Cohnhe} \mathrm{i}{ }^{2}$ ): „B. Fälle ohne freie $\mathrm{H} \mathrm{Cl}$. In diese Gruppe fällt die überwiegende Mehrzahl unserer Fälle; die Schleimhaut der Gastritiker ist besonders leicht lådirbar."

Lubarsch's 20 Stücke stammen durchwegs von Patienten mit Achylia gastrica; da ist also die oben erwăhnte Coincidenz nicht so auffallend; um so bemerkenswerther ist aber, was er über die Gewinnung von Schleimhautstückchen im Allgemeinen sagt $^{3}$ ): „Eine sehr auffăllige Eigenthümlichkeit der secretionslosen Măgen ist die grosse Vulnerabilităt ihrer Schleimhaut . . . Die Expression, bezüglich Aspiration, nach dem Probefrühstück fördert fast bei jedem mit Achylia gastrica behafteten Individuum gelegentlich ein oder mehrere abgerissene Schleimhautstïckchen zu Tage . . . . . . Seit Jahren wird in meiner Policlinik auf losgelöste Schleimhautstückchen und Gewebspartikelchen gefahndet. Die Mehrzahl der so behandelten Kranken sind nervöse Dyspeptiker

1) J. B oas. Dragnostik und Therapie der Magenkrankheiten III. Auflage S. 223.

2) 1. c. S. 285 .

3) 1. c. S. 286 . 
mit theils normalem theils übersaurem Mageninhalt. Trotz der grossen Zahl der täglich an solchen Kranken gemachten Ausspülungen finden sich Gewebspartikel bei ihnen nur relativ selten ....... Zwei Magenaffectionen sind es, die meiner Erfahrung nach in Bezug auf die mechanische Ladirbarkeit der Schleimhaut zu allen anderen in einem gewissen Gegensatze stehen: das Carcinom und die Achylia gastrica. Fast jeder, längere Zeit mit Spülung behandelte carcinomatöse Magen liefert gelegentlich ein Gewebspartikelchen und genau dasselbe ist, wie bereits gesagt, bei der Achylia gastrica der Fall."

Es darf demnach als erwiesen betrachtet werden, dass Achylia gastrica, chronische Gastritis und Carcinom, a lso gera de diejenigen Krankheitsformen, die immer, respective besonders băufig, mit Salzsauremangel einhergehen, besonders häufig Schleimbautstückchen liefern.

Wenn meine früheren Auseinandersetzungen über den fast ausschliesslich pylorischen Fundort der Darmepithelschläuche richtig sind, muss auch aus dem Wesen der mit Salzsäuremangel einhergehenden Krankheitsprocesse abgeleitet werden können, warum die beim Bestehen dieser Processe losgerissenen Stïcke aus der regio pylorica stammen; also muss auch die leichtere Vulnerabilität der Schleimhaut - nicht des ganzen Magens sondern speciell der regio pylorica - bewiesen werden. Der Beweis liegt auf der Hand, wenn man bedenkt, dass gastritische Processe, die doch auch beim Carcinom nie fehlen, constatirterweise hauptsächlich in der Pylorusregion sich abspielen; so äussern sich Kaumann ${ }^{1}$ ) und Birch Hirschfeld ${ }^{2}$ ) übereinstimmend dahin, dass sowohl bei acuter als auch bei chronischer Gastritis hauptsächlich oder ausscbliesslich die Pylorusgegend verändert ist.

So wird es also begreiflich, dass Darmepithelschläuche tragende Schleimhautstückchen, weil eben pylorischer Provenienz bei Salzsauremangel so häufig vorkommen.

1) Ka ufmann Ed u a rd. Lehrbuch der speciellen pathologischen Anatomie 1896 S. 304.

2) Birch Hirschfeld F. V. Lehrbuch der pathologischen Anatomie II. Auflage, 2. Band 1885. 
Dasselbe, was für chronische Gastritiden soeben erwiesen wurde, scheint mir auch bei Achylia gastrica der Fall zu sein. Ich kenne zwar keine Angaben, die sich auf besondere Localisation des hierbei zu Grunde liegenden pathologischen Processes in der pylorischen Region beziehen; doch spricht folgende Thatsache dafür : Ausser den sechs Darmepithelschlüuche tragenden Objecten sind es noch drei der Lubarsch' schen Stücke, die in ibrer Beschreibung die pylorische Provenienz sicher erkennen lassen (Fall 1, 3/2, 6/1) also $6+3=9$ von insgesammt 20 Stücken. Bei den übrigen 11 ist die Magenregion nicht festzustellen; sie können ebenso gut vom Fundus wie aus der pars pylorica herstammen.

Nicht so einfach und auch nicht so leicht ist der auffallende Umstand zu deuten, dass bei vierzehn Fällen Cohnh e i m's und meinen zwölf Fällen mit freier Salzsäure keine Darmepithelschläuche gefunden wurden. Es genügt nicht, zu wissen, dass die nichtcatarrhalische Schleimhaut weniger leicht lädirbar ist; ich muss auch annehmen, dass die gesunde Schleimhaut der pars pylorica noch resistenter ist, als die des ganzen übrigen Magens.

In der That zeigten meine, bei Anwesenheit freier Salzsäure losgerissene Stücke, insoferne als die Drüsenschichte vorhanden, also eine Ortsbestimmung möglich war, mit Ausnahme eines Falles, der intermediaren Gewebscharacter erkennen liess, typisches Fundusgewebe. Oder sollten die Randsäume der Epithelien im verdauungstüchtigen Magensecret untergehen, die sonst gequollenen Becherzellen aber in der freien Salzsäure verschrumpfen und dadurch die Darmepithelschläuche als solche unkenntlich werden? Ich wage nicht, es $\mathrm{zu}$ behaupten; immerhin aber besteht diese Möglichkeit, wenn auch L e uk's Befunde von Randsaumepithelien und Becherzellen bei Vorhandensein freier Salzsäure dagegen sprechen.

Das Moment der örtlich gesteigerten Vulnerabilität kommt selbstverständlich bei den Befunden, die an Operationsstücken erhoben wurden, nicht in Betracht, wohl eben ein anderes Moment: nämlich der überaus hăufige Salzsäuremangel der operirten Kranken in jenem Krankheitsstadium, wo eben die Operation bereits unvermeidlich geworden ist. So fehlt die freie Salzsäure in zehn von vierzehn Fällen $\mathrm{Hammerschlag's} \mathrm{und} \mathrm{in} \mathrm{neun}$ 
von meinen elf Fällen, wo die Schleimhautstückchen durch Gastroenterostomie, resp. Resectio pylori oder ventriculi gewonnen wurden; dass in den zehn resp. neun, also insgesammt neunzehn Fällen ohne freie Salzsăure eher Darmepithelschläuche vorkommen werden, als in den vier resp. zwei, insgesammt sechs Fällen $m$ it frei er Salzsäure, ist von vornherein wahrscheinlich, um so eher, als an meinen zwei Objecten mit freier Salzsäure und ohne Darmepithelschläuche der rein typische Funduscharacter nicht zu verkennen war (daher auch keine Darmepithelschläuche zu erwarten waren); andererseits bei meinen drei Fällen ohne freie Salzsăure und mit Darmepithelschlauchen die Drüsenelemente sicheren pylorischen Character trugen.

Diese Coincidenz von Salzsăuremangel und Darmepithelschläuchen, die auch in Hammerschlag's Fällen eine Versuchung bildet, einen tieferen Zusammenhang anzunehmen, wird durch Leuk's besprochene Fälle ihrer scheinbaren Bedeutung entkleidet; denn wir sahen ja, dass dort drei Fällen mit Salzsäure und Darmepithelschläuchen drei andere ohne Salzsäure und ohne Darmepithelschlauche gegenüberstanden.

Ich will an dieser Stelle einer Vermuthung Ausdruck geben, die mangels sachlicher Belege nur den Anspruch haben kann, als Hypothese zu gelten. Doch drăngt sich diese Vermuthung unwillkürlich auf, wenn eine Parallele zwischen den Fundorten der versprengten Darmelemente und dem häufigen Sitz von peptischen Geschwüren und Carcinomen gezogen wird. Ich brauche wohl nicht $\mathrm{zu}$ betonen, dass ich die Pylorusregion meine.

Sollten vielleicht die Randsaumepithelien, die kein schützendes schleimiges Oberende, wie die normalen Oberflächenepithelien des Magens, besitzen - eine Circulationsstörung und reichliches peptisches Secret immer vorausgesetzt -, in erster Linie angedaut werden und dadurch Veranlassung zur Bildung des peptischen Geschwüres geben?

Oder sollten die Darmepithelschläuche an solchen Stellen den Ausgangspunkt für Carcinombildung darstellen? (Siehe die alte Theorie über Neubildungen aus versprengten Keimen.) 
Sollte demnach auch das Vorkommen von peptischen Geschwüren am unteren Oesophagusende mit den daselbst befindlichen unteren cardialen Oesophagusdrüsen, resp. mit den in diesen vorkommenden Darmepithelschläuchen in Verbindung stehen?

Die Schlüsse, welche ich aus meinen Untersuchungen, sowie aus der kritischen Betrachtung der Literatur ziehen $\mathrm{zu}$ sollen glaube, lauten:

1) Die mit Darmepithel ausgekleideten Schläuche des menschlichen Magens sind keine pathologischen Gebilde.

2) Sie sind als versprengte Darmelemente anzusehen, die der Magenschleimhaut als durchaus fremdartige Bildungen eingepflanzt sind.

3) Sie kommen fast ausschliesslich in der Regio pylorica und intermedia vor; bei den grossen individuellen Schwankungen in der Ausbreitung dieser Regionen werden sie unter Umständen auch in grösserer Entfernung vom Pylorus angetroffen.

4) Sie können auch in nächster Nahe des Pylorus vollkommen fehlen.

5) Der Schleimhautbezirk, in dem sie eingesprengt sind, kann jeder pathologischen Veränderung entbelıren, aber auch die höchsten Grade interstitieller und Drüsenerkrankung zeigen.

6) Dass sie bei gewissen Krankheiten häufiger angetroffen werden als bei anderen, hängt von Umstanden ab, die beim Erlangen der betreffenden Schleimhautstücke (durch Magenschlauch oder bei Operationen) eine gewisse Rolle spielen.

Es sei mir gestattet, Herrn Professor Pertik meinen ergebensten Dank für die gütige Förderung dieser Arbeit auszusprechen. 
Ueber das normale Oberflächen-Epithel des Magens etc.

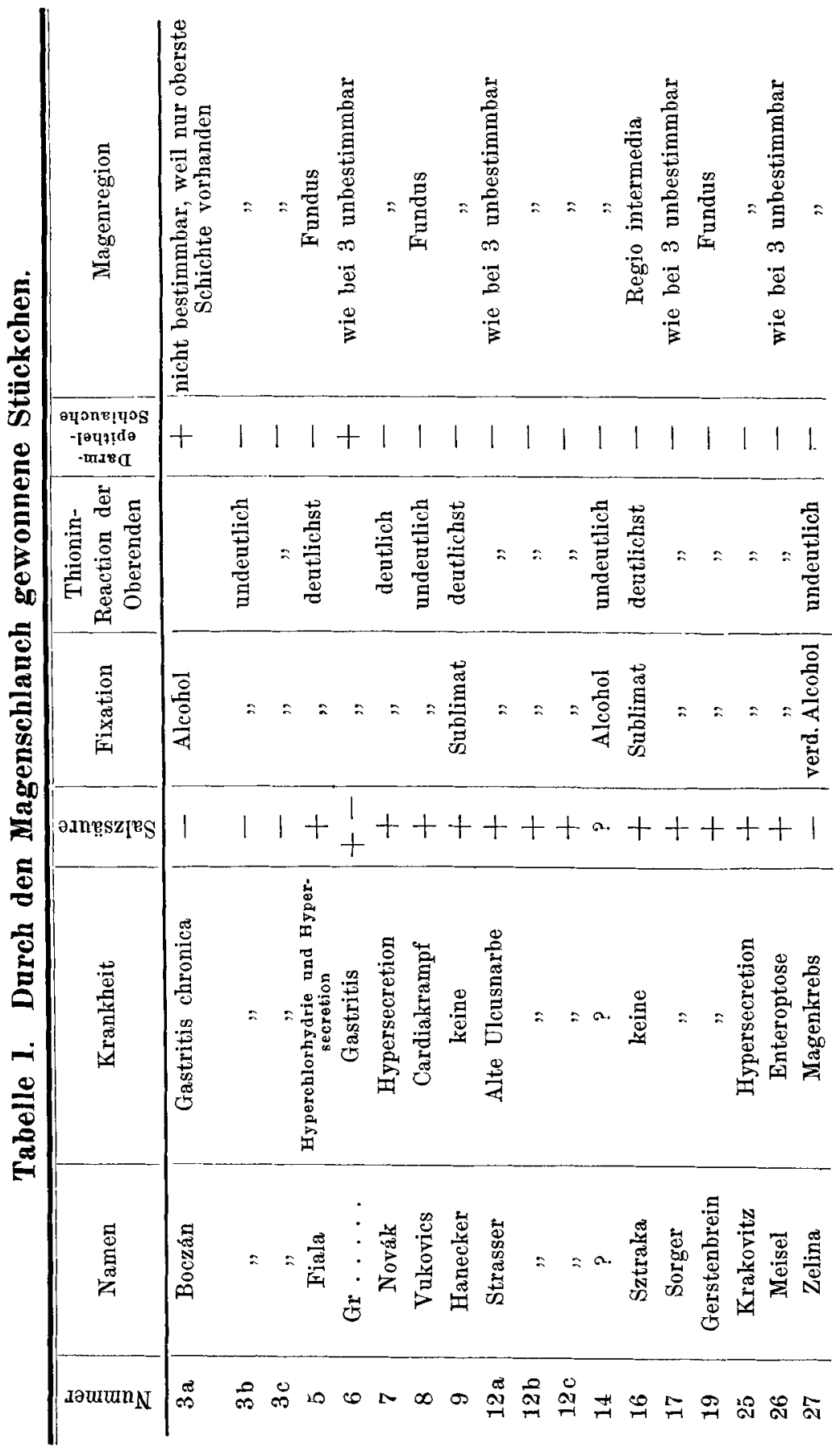




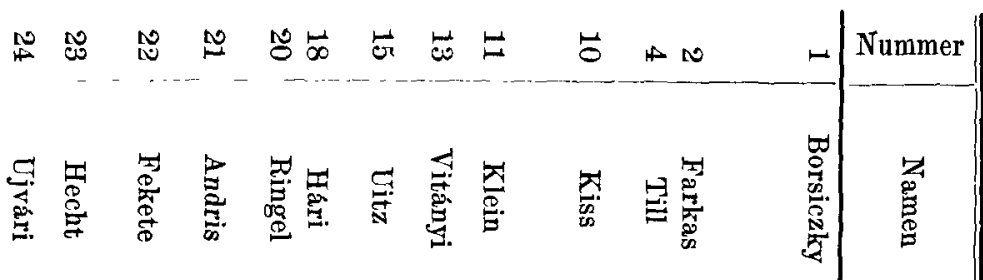

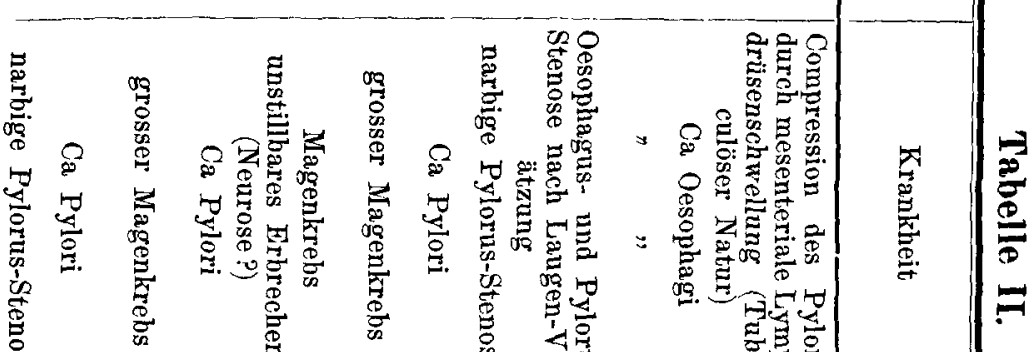

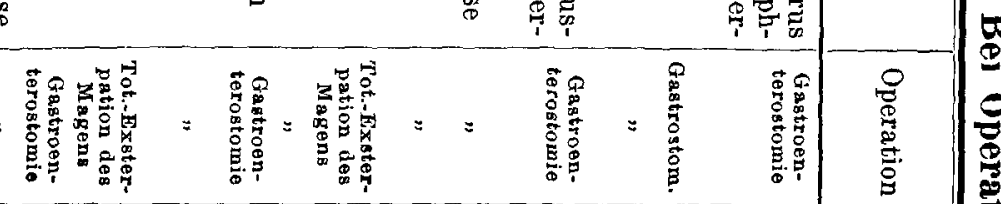

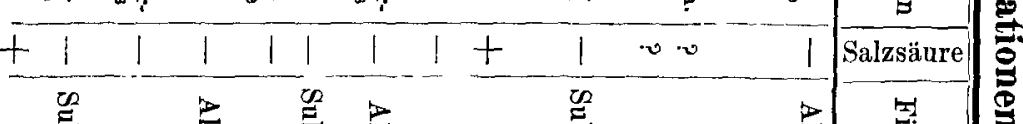

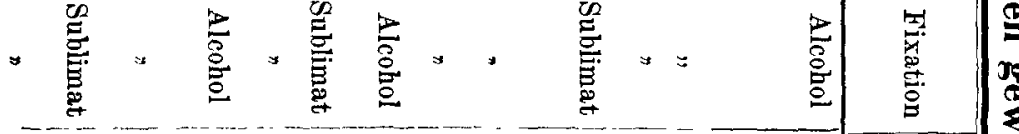

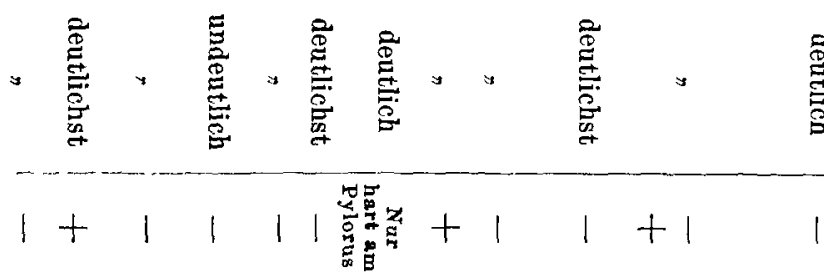

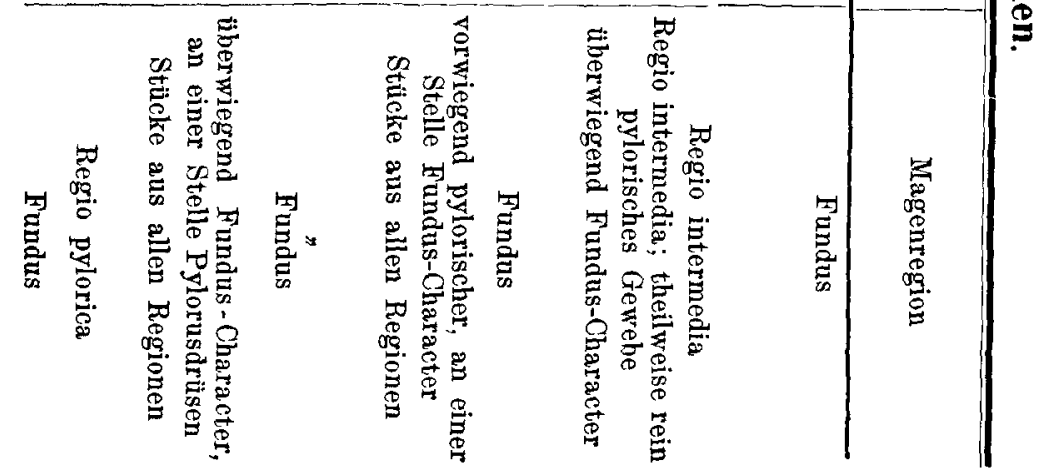




\section{Erklärung der Abbildungen auf Tafel XXXV.}

Fig. 1. Fall 5. Magen. Fixat, Alcohol. Färb. Hämatox.-Eosin. Vergr. Leitz Oc. IV. Immers. Die Zellen des Oberflächenepithels durch Alcohol verschrumpft, bilden ein leeres Vetz.

Fig. 2. Fall 9. Magen. Fixat. Sublim. Färb. Hämatox.-Eosin. Vergr. Leitz Oc. IV. Immers, Intacte Oberenden; extravasirte rothe B.-K. (offenbar beim Abreissen des Stuckchens.)

Fig. 3. Fall 10. Magen. Fixat. Sublim. Färb. modif. Thionin. Vergr. Leitz Oc. IV. Immers. Sehr grosse Oberenden, der basale Theil kaum sichtbar; Schleimpfropf mit starker Thioninreaction.

Fig. 4. Fall 1. Magen. Fixat. Alcohol. Färb. modif. Thionin. Vergr. Leitz Oc. I, Obj 3. Deutliche Schleimfärbung in den Drusenvorräumen.

Fig. 5. Fall. 5. Magen. Fixat. Alcohol. Färb. modif. Thionin. Vergr. Leitz Oc I, Obj. 3. Deutliche Schleimfärbung in den Drüsenvorräumen.

Fig. 6. Fall 2. Magen. Fixat. Alcohol. Färb, modif. Thionin. Vergr Leitz Oc. I, Obj. 7. Drusenvorräume verlängert mit deutlicher Schleimreaction.

Fig. 7. Fall 13. Magen. Fixat. Sublim. Färb. modif. Thionin. Vergr. Leitz Oc. I, Obj. 3. In der Mitte des Gesichtsfeldes Darmepithelschläuche mit roth gefärbten Becherzellen; rechts und links gleichmässig hellroth gefärbte Drusenvorräume; solitäre Follikel.

Fig. 8, 9, 10. Fall 4. Magen. Fixat. Alcohol. Färb. modif. 'Thionin. Vergr. Leitz Oc. I. Immers. Darmepithelschläuche längs und quer getroffen in adenoides Gewebe (mit spärlichen Plasmazellen) eingebettet.

Fig. 11. Fall 1. Dünndarm. Fixat. Alcohol. Färb. modif. Thionin. Vergr. Leitz Oc. I, Obj. 7. Dunndarmzotten mit Becherzellen.

Fig. 12. Fall 13, Magen Fixat. Subl. Fürb. modif. Thionin. Verg. Leitz Oc. III. Immers. Unten Pylorusdrüsen mit den characteristischen, dem Zellgrund platt angedrückten Kernen; links oben Drüsen von pylorischem Character mit einzelnen blau gefärbten Belegzellen (intermediäre Zone).

Fig. 13, Fall 5. Magen. Fixat, Alcobol. Färb. modif. Thionin. Vergr. Leitz Oc. IV. Immers. Durch Alcohol verschrumpfte Oberenden mittelst Thionin roth gefärbt. (Entsprechende Stelle von Fitg. 5 stark vergrössert.)

Fig. 14. Fall 9. Magen. Fixat. Sublim. Färb. modif. Thionin. Vergr. Leitz Oc. IV. Immers. Kleine Oberenden, Basaltheil sehr stark gefärbt.

Fig. 15. Fall 1. Magen. Fixat. Alcohol. Färb. modif. Thionin. Vergr. Leitz Oc. IV. Immers. (Eine entsprechende Stelle von Fig. 4 in starker Vergrösserung.) Grosse Oberenden.

Fig. 16. Fall 12a. Magen. Fixat. Sublim. Furb. modif. Thionin. Vergr. Leitz Oc. IV. Immers. Mittelgrosse Oberenden schwach gefärbt. Zellgrenzen an den basalen Theilen undeutlich. 
Fig. 17. Fall 4. Magen. Fixat. Alcohol. Färb. modif. Thionin. Vergr. Leitz Oc. IV. Immers. Randsaum himmelblau, Zelleiber hell graublau.

Fig. 18. Fall 13. Magen. Fixat. Sublim. Färb. Hämatox-Eosin. Vergr. Leitz Oc. IV. Immers. (Entsprechende Stelle von Fig 7 stark vergrössert.) Links Darmepithel mit Randsaum und einer Becherzelle, rechts Magenoberfl.-Epith. mit Oberenden; deutliche Stufe zwischen beiden Epithelarten.

Fig 19 Fall 1. Dunndarm. Fixat. Alcohol. Färb. modif. Thionin. Vergr. Leitz Oc. IV. Immers (Entsprechende Stelle von Fig. 11 stark vergrössert) Himmelblauer Randsaum, Zellleiber hell blaugrau; zwei roth gefärbte Becherzellen.

Fig. 20. Fall 4. Magen Fixat. Alcohol. Färb. Thionin ohne SublimatVorbehandlung. Vergr. Leitz Oc. IV. Immers. Becherzellen himmelblau, übrige Zellen violett.

Fig 21. Fall 1. Dünndarm. Fixat. Alcobol. Färb. Thionin ohne Sublimat - Vorbehandlung. Vergr. Leitz Oc. IV. Immers. Dicker Schnitt, daher viele theilweise übereinander gelagerte Zellkerne in mehreren Reiben. Becherzellen himmelblau, ubrige Zellen violett.

Fig. 22. Oberflächenepithel aus dem IIagen von Raja asterias (aus Oppel 1. c. S. 36). 